\title{
Strong Field Control of the Interatomic Coulombic Decay Process in Quantum Dots
}

\author{
Anika Haller \\ Institute of Methods for Material Development, Helmholtz-Zentrum Berlin für \\ Materialien und Energie GmbH, Albert-Einstein-Str. 15, 12489 Berlin, Germany \\ Ying-Chih Chiang \\ The Chinese University of Hong Kong, Sha Tin, N.T., Hong Kong \\ Theoretische Chemie, Physikalisch-Chemisches Institut, Im Neuenheimer Feld 229, \\ 69120 Heidelberg, Germany \\ Maximilian Menger \\ Dipartimento di Chimica e Chimica Industriale, University of Pisa, Via Giuseppe \\ Moruzzi 13, 56124 Pisa, Italy \\ Theoretische Chemie, Physikalisch-Chemisches Institut, Im Neuenheimer Feld 229, \\ 69120 Heidelberg, Germany \\ Emad F. Aziz \\ Institute of Methods for Material Development, Helmholtz-Zentrum Berlin für \\ Materialien und Energie GmbH, Albert-Einstein-Str. 15, 12489 Berlin, Germany \\ Department of Physics, Freie Universität Berlin, Arnimallee 14, 14195 Berlin, Germany \\ School of Chemistry, Monash University, Clayton 3800, VIC, Australia
}

Annika Bande

Institute of Methods for Material Development, Helmholtz-Zentrum Berlin für

Materialien und Energie GmbH, Albert-Einstein-Str. 15, 12489 Berlin, Germany

Theoretische Chemie, Physikalisch-Chemisches Institut, Im Neuenheimer Feld 229, 69120 Heidelberg, Germany

\section{Abstract}

Email address: annika.bande@helmholtz-berlin.de (Annika Bande) 
In recent years the laser-induced interatomic Coulombic decay (ICD) process in paired quantum dots has been predicted [J. Chem. Phys. 138 (2013) 214104]. In this work we target the enhancement of ICD by scanning over a range of strong-field laser intensities. The GaAs quantum dots are modeled by a one-dimensional double-well potential in which simulations are done with the space-resolved multi-configuration time-dependent Hartree method including antisymmetrization to account for the fermions. As a novelty a complementary state-resolved ansatz is developed to consolidate the interpretation of transient state populations, widths obtained for the ICD and the competing direct ionization channel, and Fano peak profiles in the photoelectron spectra. The major results are that multi-photon processes are unimportant even for the strongest fields. Further, below- $\pi$ to $\pi$ pulses display the highest ICD efficiency while the direct ionization becomes less dominant. Keywords: Electron Dynamics, Laser Control, Interatomic Coulombic Decay, Quantum Dot, Strong Field

\section{Introduction}

"ICD appears everywhere!" [1] - this slogan summarizes the nearly twenty years of success history of the ultrafast interatomic (intermolecular) Coulombic decay (ICD) process from its first theoretical prediction by Cederbaum, Zobeley, and Tarantelli [2] to its widespread theoretical and experimental observation $[3,4]$ in various atomic and molecular systems including clusters of noble gas atoms $[5,6,7,8]$, endohedral fullerenes $[9,10,11]$, aqueous solutions $[12,13,14,15,16,17]$, biological systems [18, 19, 20, 21, 22, 23], and nanomaterials $[24,25,26]$ just to name a few. 
ICD can be understood as a delocalized Auger decay over two or more atomic species which is mediated by the long-range Coulomb force among electrons residing on the different sites: on one site a high-energy electron relaxes into a lower-energy state and transfers its energy to another electron on one of the neighboring sites which is then ionized. The decaying resonance excited state is typically prepared by either inner-valence ionization [3, 4, 27], resonant excitation $[28,29,30,31]$, electron $[4,32,33,34,35,36]$, or ion impact $[4,37]$.

Moreover, the above slogan directs into the future. It motivates the interdisciplinary community of ICD researchers to push the frontiers towards a comprehensive fundamental understanding of ICD as well as towards novel materials. Our contributions support both directions. Firstly, we pioneered material sciences investigations of ICD by studying pairs of nano-structured semiconductors, namely quantum dots (QD) [24, 25, 38]. They are available from various fabrications techniques as self-assembled [39], nanowire [40], etched vertical [41], and gated two-dimensional electron gas [42] quantum dots. Such materials are attractive candidates for a device application of ICD in the field of energy conversion, e.g. as next-generation infrared photo detector or solar cell. This is because the QD pair's electronic structure, and hence the ICD performance, can be custom made through geometry control of the QDs' distance [25, 38] (this holds likewise for quantum wells [26]), widths [43], and heights [43, 44].

Secondly, we explicitly solve the time-dependent electronic Schrödinger equation in a space-resolved fashion $[25,34]$ for the scenario of two correlated electrons. This renders insight into the transient electronic level occupations 
during ICD in addition to the decay rates which can likewise be obtained from non-hermitian electronic structure theory for resonances $[6,27,45,46]$ and nuclear dynamics of the cluster explosion after ICD [47, 48, 49]. In cases lessaccurate partially-correlated electron dynamics has been used in combination with hole [50] or nuclear [17] dynamics. In this paper we push the theory to another level in solving the time-dependent electronic Schrödinger equation in an alternative state-resolved ansatz to consolidate and interpret our spaceresolved results.

Thirdly, we explicitly consider the radiative initiation of ICD in our description [51]. Control of ICD by field strength variation is the theme of this paper. Before we lay out the details, let us introduce the two-state-andcontinuum model system of the paired QDs (Fig. 1). Each QD is represented by an electron binding potential where the two levels $L_{0}$ and $L_{1}$ of the left and the single level $R_{0}$ of the right QD are in the energetic order $E_{L_{0}}^{1 e}<E_{R_{0}}^{1 e}<E_{L_{1}}^{1 e}$. The system is initially in its $\left|L_{0} R_{0}\right\rangle$ ground state with one electron in the lowest level of each QD. A laser excites the electron in the left $\mathrm{QD}$ such that the two-electron resonance state $\left|L_{1} R_{0}\right\rangle$ is populated. This initiates ICD where the $L_{1}$ electron relaxes to the $L_{0}$ level again while energy becomes available to ionize the right binding potential through exciting the $R_{0}$ electron into the electronic continuum with energies $\varepsilon$, i.e. into the state manifold $\left|L_{0} \varepsilon\right\rangle[25,51]$. States with both electrons localized in the same QD were found to be irrelevant for the processes investigated [25, 38]. Note as well that available paired QDs meeting this theoretical description are singly-charged e.g. by electron transport from a reservoir [39, 40, 41, 42]. Either a triplet state, which is stable over $100 \mathrm{~ns}[40,41]$ or the energetically 


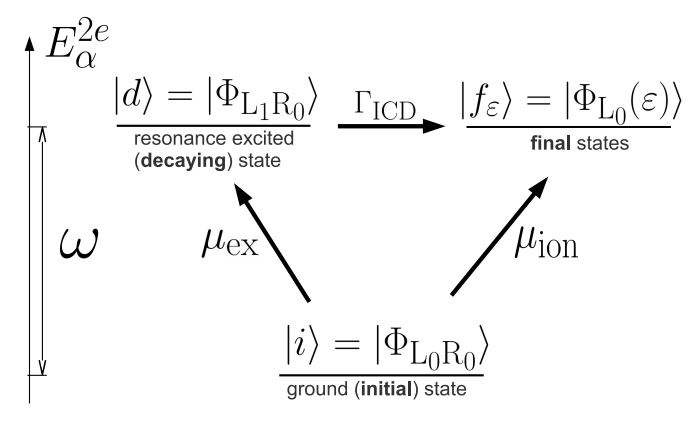

Figure 1: Schematic representation of the relevant two-electron states in the paired QD. The $\left|L_{0} R_{0}\right\rangle$ ground state is by $\omega$ lower in energy than the equally-energetic decaying and continuum states $\left|L_{1} R_{0}\right\rangle$ and $\left|L_{0} \varepsilon\right\rangle$. These states can be addressed by the laser, the first through resonant excitation (ex), the second through direct ionization (ion). $\left|L_{1} R_{0}\right\rangle$ can decay into $\left|L_{0} \varepsilon\right\rangle$ via ICD.

favored singlet state could be established for the full duration of ICD and likewise be calculated $[25,51]$.

When speaking of laser control of ICD an efficient preparation of the decaying resonance state is anticipated. One control parameter is the laser focus that influences the direct ionization of the $R_{0}$ electron [51]. A few others, which will be subject to future publications, are off-resonance lasing actions, polarization effects relevant to other typical QD geometries, and pulse duration. Their combination may eventually be cast into an optimal control scheme.

In this study we investigate the influence of different strengths of a pulsed infrared-laser field on the ICD process in QDs. They are in the range of $3 \cdot 10^{7}-2 \cdot 10^{9} \mathrm{~W} / \mathrm{cm}^{2}[52,53]$ and thus lie well below the maximum intensities $10^{18} \mathrm{~W} / \mathrm{cm}^{2}$ typically accessible in regular laser labs for a variety of energies from ultraviolet to infrared pulses [54]. To date experiments on ICD have only 
been done for atomic and molecular clusters and require soft X-ray pulses of similar intensity that are only available in large-scale synchrotron facilities as e.g. BESSY II $[28,55]$. There the peak intensity is prescribed by the beamline used and typically allows for a sub- $\pi$ pulse excitation only. Hence, to our knowledge, ICD has experimentally never been investigated with view on laser strengths. For atoms and molecules this may become possible at high-brilliance soft X-ray sources specifically prepared for intensity variation, or when laser technology advances towards generating soft X-ray pulses in regular laser labs. At that time our theoretical method will be ready to accompany such experiments. By contrast, no obvious technical obstacle may hinder a potential QD ICD lab experiment on the influence of infrared lasing strength, and indeed QD experiments have already been done on population inverting Rabi oscillations with ultraviolet to visible light in the intensity range $10^{3}-10^{6} \mathrm{~W} / \mathrm{cm}^{2}[56,57]$. But there photoionization was no relevant process.

Although not ICD, the Auger decay after core-ionization of atoms has been studied in competition with photoionization as function of the strength of hard X-ray fields with $10^{14}-10^{19} \mathrm{~W} / \mathrm{cm}^{2}[53,58]$. In focus was the field strength dependence of the shape of the electron spectrum after irradiation with time-symmetric $n \pi$ pulses which established an $n$-fold multiplet structure. Such a profile can be observed when the decay is much faster then the pulse, i.e. where the spectral widths of a $\pi$ pulse, or the Rabi oscillation time of any $n \pi$ pulse, is significantly larger than the decay widths [53, 58]. In the inverse case $[51,59,60,61]$ the photoelectron peak establishes an ideal Fano profile when no strong-field bending of continuum states occurs [60]. 
In our previous work we have been able to deduce the Fano rather than the multiplet regime and showed there a nearly ideal Fano profile for an $n=1 \pi$ pulse [51]. Here we will extend these findings towards higher- $n \pi$ pulses.

The course of the manuscript is this: In Sec. 2.1 we introduce the model for the QD pair followed by the two theoretical methods for space- and a state-resolved electron-dynamics calculations (Secs. 2.2.1 and 2.2.2). To compare both representations we begin discussing the results with the known $\pi$-pulse-induced ICD process (Sec. 3.1) and then turn to stronger $n \pi$-pulses (Sec. 3.2.1) as well as arbitrarily strong (Sec. 3.2.2) and weak fields (Sec. $3.2 .3)$.

\section{Theory}

\subsection{Paired Quantum Dot Model}

The pair of QDs in which we investigate ICD is modeled by two onedimensional negative Gaussian potentials expanding into the $z$-direction,

$$
\hat{V}_{\mathrm{QD}}(z)=-D_{L} e^{-b_{L}(z+\tilde{R} / 2)^{2}}-D_{R} e^{-b_{R}(z-\tilde{R} / 2)^{2}},
$$

where $\tilde{R}=8.0$ a.u. $(86.68 \mathrm{~nm})$ is the distance between the potential minima, $D_{L}=1.0$ a.u. (10.30 meV) and $D_{R}=0.8$ a.u. (8.24 meV) are the depths of the left $(\mathrm{L})$ and right $(\mathrm{R})$ potential wells. With $b_{L}=0.25$ a.u. and $b_{R}=1.0$ a.u. we determine the corresponding full width at half maximum of the two potentials via $r_{L, R}=2 \sqrt{\ln 2 / b_{L, R}}$ which gives $r_{L}=36.08 \mathrm{~nm}$ and $r_{R}=18.04$ nm. Note that our calculations were performed in atomic units. The numerical data in this paper is given in units of GaAs QDs (nm for distances, meV for energies) with a material specific effective mass and dielectric constant, 
$m^{*}=0.063$ and $\kappa=12.9$ [62], and the conversion equations as in previous work [43]. The parameters fulfill the requirement of keeping the spatial overlap of the Gaussian potential wells' bound one-particle states negligible, so that two-electron wavefunctions can be approximated by an antisymmetrized Hartree product (see below).

The model is based on our previous calculations in a quasi-one dimensional system, i.e. in three-dimensional space with only one-dimensional continuum $[25,51]$ for which we showed in other works that a simplification into a true one-dimensional potential is allowed [36, 38].

The single-electron eigenstates of the Hamiltonian for the $i$-th electron,

$$
\hat{h}_{i}=-\frac{1}{2} \frac{\partial^{2}}{\partial z_{i}^{2}}+\hat{V}_{\mathrm{QD}}\left(z_{i}\right)
$$

fulfill the time-independent Schrödinger equation for discrete energies labeled by $n$,

$$
\hat{h}_{i}\left|\phi_{n}\right\rangle=E_{n}^{1 e}\left|\phi_{n}\right\rangle
$$

and for continuous energies labeled by $\varepsilon$,

$$
\hat{h}_{i}|\phi(\varepsilon)\rangle=\varepsilon|\phi(\varepsilon)\rangle \text {. }
$$

There are two bound states for the left $\left(\left|\phi_{L_{0}}\right\rangle,\left|\phi_{L_{1}}\right\rangle\right)$ and one for the right well $\left(\left|\phi_{R_{0}}\right\rangle\right)$ with the following order of the energies $E_{L_{0}}^{1 e}<E_{R_{0}}^{1 e}<E_{L_{1}}^{1 e}$. Above these energies the spectrum is continuous.

The two-electron Hamiltonian,

$$
\hat{H}_{\mathrm{el}}=\hat{h}_{1}+\hat{h}_{2}+\hat{V}_{\mathrm{Coul}}\left(z_{12}\right) \text {, }
$$


contains, besides the one-electron Hamiltonians of Eq. (2), a term for the Coulomb interaction, namely

$$
\hat{V}_{\text {Coul }}\left(z_{12}\right)=\sqrt{\frac{\pi}{2}} e^{z_{12}^{2} / 2} \operatorname{erfc}\left(z_{12} / \sqrt{2}\right)
$$

with $z_{12}=\left|z_{1}-z_{2}\right|$. It accounts for the transition of the Coulomb interaction defined as $r_{12}^{-1}=\left|\mathbf{r}_{1}-\mathbf{r}_{2}\right|^{-1}$ in three-dimensional systems into one dimension here $[36,38]$.

The solution to the respective time-independent Schrödinger equation,

$$
\hat{H}_{\mathrm{el}}\left|\Phi_{\alpha}\right\rangle=E_{\alpha}^{2 e}\left|\Phi_{\alpha}\right\rangle
$$

yields the two-electron eigenstates $\left|\Phi_{\alpha}\right\rangle$ with the corresponding eigenenergies $E_{\alpha}^{2 e}$. The index $\alpha$ denotes the possible configurations of the two-electron system. There one has to consider the three different cases of either both electrons occupying bound QD levels, both occupying continuum levels, or a mixture of both. Note particularly that the all-bound two-electron states relevant to ICD localize one electron in each of the potentials, although for triplets one and for singlets four states with both electrons in the same potential exists which are known to not disturb the dynamics. [25, 38]

Here we deliberately chose the more-intensely investigated triplet electron configuration. The antisymmetrized two-electron eigenstates can be approximated via two-term products of single-particle solutions,

$$
\left|\Phi_{\alpha}\right\rangle=\left\{\begin{array}{l}
\left|\Phi_{n n^{\prime}}\right\rangle=\frac{1}{\sqrt{2}}\left(\left|\phi_{n^{\prime}}\right\rangle\left|\phi_{n}\right\rangle-\left|\phi_{n}\right\rangle\left|\phi_{n^{\prime}}\right\rangle\right) \\
\left|\Phi_{n}(\varepsilon)\right\rangle=\frac{1}{\sqrt{2}}\left(|\phi(\varepsilon)\rangle\left|\phi_{n}\right\rangle-\left|\phi_{n}\right\rangle|\phi(\varepsilon)\rangle\right) \\
\left|\Phi\left(\varepsilon, \varepsilon^{\prime}\right)\right\rangle=\frac{1}{\sqrt{2}}\left(\left|\phi\left(\varepsilon^{\prime}\right)\right\rangle|\phi(\varepsilon)\rangle-|\phi(\varepsilon)\rangle\left|\phi\left(\varepsilon^{\prime}\right)\right\rangle\right)
\end{array}\right.
$$


with the corresponding energies,

$$
E_{\alpha}^{2 e}=\left\{\begin{array}{l}
E_{n n^{\prime}}^{2 e}=E_{n}^{1 e}+E_{n^{\prime}}^{1 e}+\tilde{V}_{\mathrm{Coul}}^{n n^{\prime}} \\
E_{n \varepsilon}^{2 e}=E_{n}^{1 e}+\varepsilon+\tilde{V}_{\mathrm{Coul}}^{n \varepsilon} \\
E_{\varepsilon \varepsilon^{\prime}}^{2 e}=\varepsilon+\varepsilon^{\prime}+\tilde{V}_{\mathrm{Coul}}^{\varepsilon \varepsilon^{\prime}},
\end{array}\right.
$$

with $n, n^{\prime}=L_{0}, L_{1}, R_{0}$. $\tilde{V}_{\text {Coul }}^{\alpha}$ denotes the expectation value of the Coulomb energy which is approximately $\tilde{V}_{\text {Coul }}^{\alpha} \approx 1 / \tilde{R}$ for the two relevant $n n^{\prime}$-cases, $\left|L_{0} R_{0}\right\rangle$ and $\left|L_{1} R_{0}\right\rangle$, but significantly smaller for interactions including continuum electrons.

\subsection{Electron Dynamics}

As we continue our electron-dynamics study of ICD in a QD pair initiated by lasers [51] by now including pulse variations, we aim at the solution to the time-dependent Schrödinger equation,

$$
i \frac{\partial}{\partial t}|\Psi(t)\rangle=\hat{H}(t)|\Psi(t)\rangle
$$

The interaction of the electrons with the electromagnetic field is described by a time-dependent term $\hat{H}_{\mathrm{em}}(t)$ in the Hamiltonian,

$$
\hat{H}(t)=\hat{H}_{\mathrm{el}}+\hat{H}_{\mathrm{em}}(t)
$$

Within the semiclassical dipole approximation it is represented by

$$
\hat{H}_{\mathrm{em}}(t)=-\mathcal{E}(t) \cdot \hat{\boldsymbol{\mu}}
$$

with the electric dipole operator $\hat{\boldsymbol{\mu}}=-\sum_{i} \hat{z}_{i} \mathbf{e}_{z}$ summing over the coordinates of negatively charged electrons. The time-dependent electric field $\mathcal{E}(t)=$ $\mathcal{E}(t) \mathbf{e}_{z}$ is linearly polarized into $z$-direction, hence

$$
\hat{H}_{\mathrm{em}}(t)=\mathcal{E}(t)\left(\hat{z}_{1}+\hat{z}_{2}\right) .
$$


Due to the limited minimal focus of a typical laser the complete paired QD system is resonantly excited via an $n \pi$-laser pulse with the electric field,

$$
\mathcal{E}(t)=n \varepsilon_{0} \cos (\omega t) \sin ^{2}\left(\pi \frac{t}{t_{w}}\right) \Theta\left(t_{w}-t\right) .
$$

The field strength $\varepsilon_{0}=2 \omega$ is determined for the $\pi$-pulse case $n=1$ [51, 63], where $\omega=E_{L_{1} R_{0}}^{2 e}-E_{L_{0} R_{0}}^{2 e}$ is the resonant excitation energy. For $n=1$ the fixed pulse duration $t_{w}=2 \pi / \varepsilon_{0}$ allows exactly for a single $\left|L_{0} R_{0}\right\rangle \rightarrow\left|L_{1} R_{0}\right\rangle$ inversion, otherwise for $n$ inversions. Here, we also study a variety of strong pulses with $n>1, n \in \mathbb{N}$ as well as weak pulses with $0<n<1, n \in \mathbb{R}$ and observe the decay of the $\left|L_{1} R_{0}\right\rangle$ state with the ICD rate $\Gamma_{\mathrm{L}_{1} R_{0}}=\Gamma_{\mathrm{ICD}}$. The intensity of the laser field relates to the field strength via $I=\left(n \varepsilon_{0}\right)^{2} /(8 \pi \alpha)$ (in atomic units) with the fine structure constant $\alpha$ as inverse of the speed of light in vacuum.

In the following, we introduce two representations of the time-dependent wavefunction as solution to the Schrödinger equation (10).

\subsubsection{Space-Resolved Representation}

In a space-resolved calculation the real-time dynamics is calculated within an interval in which the wavefunction propagates. During the propagation, the initial wavepacket interacts with an external laser pulse and undergoes simultaneously ICD and a direct photoionization processes. For this purpose we make use of the multi-configuration time-dependent Hartree (MCTDH) method [64, 65] implemented in the Heidelberg MCTDH package $[66,67]$. The spatial wavefunction is represented by a sum over Hartree products with time-dependent coefficients $A_{j_{1} \ldots j_{k}}(t)$ and likewise time-dependent single-particle functions (SPFs) $\psi_{j_{i}}\left(\mathbf{r}_{i}, t\right)$. In this representation, the total 
electronic wavefunction is resolved in space, instead of the individual states, so that the continuum is treated at equally high accuracy as the bound states. Yet, the information of the population of a specific bound state is still available via projections (see below). For our two-electron system with one degree of freedom $z_{i}$ per electron the wavefunction looks as follows,

$$
\Psi^{\mathrm{MCTDH}}\left(z_{1}, z_{2}, t\right)=\sum_{j_{1}}^{n_{1}} \sum_{j_{2}}^{n_{2}} A_{j_{1} j_{2}}(t) \psi_{j_{1}}^{(1)}\left(z_{1}, t\right) \psi_{j_{2}}^{(2)}\left(z_{2}, t\right) .
$$

Both, the time-dependent SPFs and the expansion coefficients are propagated according to the MCTDH equations of motion, which are derived from the Dirac-Frenkel time-dependent variational principle, i.e. $\left\langle\delta \Psi\left|H-i \partial_{t}\right| \Psi\right\rangle=0$. In a preceding block improved relaxation, i.e. a propagation in negative imaginary time with $\hat{H}=\hat{H}_{\mathrm{el}}$, we use that at $t=0$ the SPFs of the wavefunction correspond to the time-independent one-electron eigenfunctions $\phi_{n}\left(z_{i}\right)$ for both electrons in their respective well's ground state, as

$$
\Psi^{\mathrm{MCTDH}}\left(\mathbf{r}_{1}, \mathbf{r}_{2}, t=0\right)=2^{-1 / 2}\left(\phi_{L_{0}}\left(z_{1}\right) \phi_{R_{0}}\left(z_{2}\right)-\phi_{R_{0}}\left(z_{1}\right) \phi_{L_{0}}\left(z_{2}\right)\right)
$$

For spin triplet states the spatial wavefunction is antisymmetrized by imposing the condition $A_{j_{1} j_{2}}(t)=-A_{j_{2} j_{1}}(t)$. From the relaxation we find the initial state $\Phi_{L_{0} R_{0}}\left(z_{1}, z_{2}\right)$ and the resonance excited state $\Phi_{L_{1} R_{0}}\left(z_{1}, z_{2}\right)$. The former is then propagated in real time to obtain the time-dependent wavefunction. For the SPFs the Runge-Kutta integrator of order 8 was applied, for the A coefficients a short-time Lanczos integrator (SIL). The effectively realized propagation time step was about 0.0639 ps for state populations and 0.0064 ps for electron spectra, respectively.

For result analysis, we determine the populations of the single-electron states, which can be calculated via the projections of the single-particle states 
on the two-particle time-dependent wavefunction. For the inclusion of the second coordinate we need to project on the two-particle identity first,

$$
\begin{aligned}
\mathbb{1}= & \frac{1}{2} \sum_{n n^{\prime}}\left|\Phi_{n n^{\prime}}\right\rangle\left\langle\Phi_{n n^{\prime}}\left|+\sum_{n} \int d \varepsilon\right| \Phi_{n}(\varepsilon)\right\rangle\left\langle\Phi_{n}(\varepsilon)\right| \\
& +\frac{1}{2} \iint d \varepsilon d \varepsilon^{\prime}\left|\Phi\left(\varepsilon, \varepsilon^{\prime}\right)\right\rangle\left\langle\Phi\left(\varepsilon, \varepsilon^{\prime}\right)\right| .
\end{aligned}
$$

The populations of the discrete one-electron states $\left|\phi_{n}\right\rangle$ as well as the continuum states $|\phi(\varepsilon)\rangle$ are then calculated as

$$
\begin{aligned}
P_{n}(t) & =2\left|\left\langle\phi_{n}|\mathbb{1}| \Psi(t)\right\rangle\right|^{2} \\
& =\sum_{n^{\prime}}\left|\left\langle\Phi_{n^{\prime} n} \mid \Psi(t)\right\rangle\right|^{2}+\int d \varepsilon\left|\left\langle\Phi_{n}(\varepsilon) \mid \Psi(t)\right\rangle\right|^{2} \\
P(t, \varepsilon) & =2|\langle\phi(\varepsilon)|\mathbb{1}| \Psi(t)\rangle|^{2} \\
& =\sum_{n}\left|\left\langle\Phi_{n}(\varepsilon) \mid \Psi(t)\right\rangle\right|^{2}+\int d \varepsilon^{\prime}\left|\left\langle\Phi\left(\varepsilon, \varepsilon^{\prime}\right) \mid \Psi(t)\right\rangle\right|^{2},
\end{aligned}
$$

where the prefactor of two accounts for two identical electrons. The populations of the two-electron states (Eq. (8)) can be obtained from the squared absolute value of the projections on the time-dependent wave function, or crosscorrelation functions $c(t)$,

$$
P_{\alpha}(t)=\left|\left\langle\Phi_{\alpha} \mid \Psi(t)\right\rangle\right|^{2}=\left|c_{\alpha}(t)\right|^{2}
$$

After the termination of the pulse the population of $\left|\Phi_{L_{1} R_{0}}\right\rangle$ follows an exponential decay $P_{L_{1} R_{0}}(t) \propto e^{-\Gamma_{\mathrm{ICD}} t}$ with the ICD rate $\Gamma_{\mathrm{ICD}}$.

After a sufficiently long propagation time $T, \Gamma_{\text {ICD }}$ can likewise be deduced from the electron spectrum $[51,66]$,

$$
\sigma_{\alpha}(E)=2 \int_{0}^{T} \Re\left(c_{\alpha}(t) e^{i\left(E+E_{\alpha}\right) t}\right) d t
$$


around the relevant state $|\alpha\rangle=\left|\Phi_{L_{1} R_{0}}\right\rangle$ when fitting the general Fano line shape

$$
\sigma_{L_{1} R_{0}}^{\text {Fano }}(E)=\frac{1}{1+q^{2}} \frac{\left(q \cdot \Gamma_{\mathrm{ICD}} / 2+\left(E-E_{L_{1} R_{0}}\right)\right)^{2}}{\left(\Gamma_{\mathrm{ICD}} / 2\right)^{2}+\left(E-E_{L_{1} R_{0}}\right)^{2}}
$$

onto the spectrum, which is centered at the resonance position $E=E_{L_{1} R_{0}}$ and scaled with $\left(1+q^{2}\right)^{-1}$ to only take values in $[0,1]$. Moreover, the Fano profile parameter $q$ is obtained, which determines the relative importance of the ICD decay process compared to direct ionization.

Technically, in MCTDH wavefunctions and operators are described by a discrete variable representation (DVR), which is here done with 140 and 980 sine DVR points in $z$-direction in the interval [-541.78 nm, $541.78 \mathrm{~nm}$ ] and $[-3792.42 \mathrm{~nm}, 3792.42 \mathrm{~nm}]$, respectively. The grid density was $\Delta x=$ $7.74 \mathrm{~nm}$ in both cases that allows represent continuum wavefunctions with the electron having a maximum kinetic energy of $T_{\max }=99.6 \mathrm{meV}$ which corresponds to an 18-photon excitation. For the calculations on the short grid a complex absorbing potential (CAP) $[25,51]$ of $4 t h$-order and strength $\eta=8.6997 \cdot 10^{-6}$ at $z= \pm 325.07 \mathrm{~nm}$ removes the emitted electron and thus prevents its reflection at the end of the grid. The long grid is CAP free, as we can reasonably observe ICD before backscattered electrons start to penetrate the QDs geometry.

The effective Coulomb potential (Eq. (6)) has been converted into MCTDH form, a sum of products of single particle potentials, by employment of the POTFIT program $[66,68,69]$. For this conversion to be accurate, i.e. having a root mean square deviation of about $10^{-12} \mathrm{meV}$ among the exact and the fitted potential and, more importantly, not changing the results of the electron dynamics, an exact expansion over all single-particle turned out to be 
mandatory. On the comparably small grid this is, however, not time-critical (for details see Ref. [43]).

\subsubsection{State-Resolved Representation}

In a second approach we use a state-resolved representation of the system $[70,71]$. This approach is based on the two relevant two-electron eigenstates $\left|L_{0} R_{0}\right\rangle,\left|L_{1} R_{0}\right\rangle$ and the continuum $\left|L_{0} \varepsilon\right\rangle$ (cf. Fig. 1). This is a much faster way of obtaining information on the systems' dynamical behavior, but therefore we will neglect multi-photon processes. If this is legal to do, we will see by comparing the outcome with the space-resolved calculations.

The total wavefunction $|\Psi(t)\rangle$ is expanded in the orthonormal basis of the time-independent two-electron eigenstates Eq. (8). By making use of the completeness of the basis, $|\Psi(t)\rangle$ can be multiplied by the identity (Eq. (17)) which gives

$$
\begin{aligned}
|\Psi(t)\rangle= & \frac{1}{2} \sum_{n n^{\prime}} a_{n n^{\prime}}(t)\left|\Phi_{n n^{\prime}}\right\rangle+\sum_{n} \int d \varepsilon a_{n}(t, \varepsilon)\left|\Phi_{n}(\varepsilon)\right\rangle \\
& +\frac{1}{2} \iint d \varepsilon d \varepsilon^{\prime} a\left(t, \varepsilon, \varepsilon^{\prime}\right)\left|\Phi\left(\varepsilon, \varepsilon^{\prime}\right)\right\rangle .
\end{aligned}
$$

Here, we introduced the time-dependent coefficients $a_{n n^{\prime}}(t)=\left\langle\Phi_{n n^{\prime}} \mid \Psi(t)\right\rangle$, $a_{n}(t, \varepsilon)=\left\langle\Phi_{n}(\varepsilon) \mid \Psi(t)\right\rangle$ and $a\left(t, \varepsilon, \varepsilon^{\prime}\right)=\left\langle\Phi\left(\varepsilon, \varepsilon^{\prime}\right) \mid \Psi(t)\right\rangle$. Let us consider only single-photon excitations here, i.e. besides $\left|\Phi_{L_{0} R_{0}}\right\rangle$ only the states $\left|\Phi_{L_{1} R_{0}}\right\rangle$ and $\left|\Phi_{L_{0}}(\varepsilon)\right\rangle$ are populated after applying a laser pulse (cf. Fig. 1), where $E_{L_{1} R_{0}}^{2 e}=E_{L_{0} \varepsilon}^{2 e}$. We also rename the initial state $\left|\Phi_{L_{0} R_{0}}\right\rangle=|i\rangle$, the decaying state $\left|\Phi_{L_{1} R_{0}}\right\rangle=|d\rangle$ and the final state $\left|\Phi_{L_{0}}(\varepsilon)\right\rangle=\left|f_{\varepsilon}\right\rangle$. The wavefunction of Eq. (25) then simplifies to

$$
|\Psi(t)\rangle=a_{i}(t)|i\rangle+a_{d}(t)|d\rangle+\int d \varepsilon a_{f}(t, \varepsilon)\left|f_{\varepsilon}\right\rangle .
$$


Inserting it into the time-dependent Schrödinger equation (10) gives,

$$
\begin{array}{r}
i \dot{a}_{i}(t)|i\rangle+i \dot{a}_{d}(t)|d\rangle+i \int d \varepsilon \dot{a}_{f}(t, \varepsilon)\left|f_{\varepsilon}\right\rangle= \\
a_{i}(t) \hat{H}(t)|i\rangle+a_{d}(t) \hat{H}(t)|d\rangle+\int d \varepsilon a_{f}(t, \varepsilon) \hat{H}(t)\left|f_{\varepsilon}\right\rangle,
\end{array}
$$

where $\dot{a}$ denotes the time-derivative of $a$. Eq. (27) multiplied from the left separately with $\langle i|,\langle d|$ and $\left\langle f_{\varepsilon}\right|$ gives the following set of equations,

$$
\begin{aligned}
i \dot{a}_{i}(t) & =\langle i|\hat{H}| i\rangle a_{i}(t)+\langle i|\hat{H}| d\rangle a_{d}(t)+\int d \varepsilon\left\langle i|\hat{H}| f_{\varepsilon}\right\rangle a_{f}(t, \varepsilon) \\
& =E_{i} a_{i}(t)+\mu_{\mathrm{ex}}^{*} \mathcal{E}^{*}(t) a_{d}(t)+\mathcal{E}^{*}(t) \int d \varepsilon \mu_{\mathrm{ion}}^{*}(\varepsilon) a_{f}(t, \varepsilon) \\
i \dot{a}_{d}(t) & =\langle d|\hat{H}| i\rangle a_{i}(t)+\langle d|\hat{H}| d\rangle a_{d}(t)+\int d \varepsilon\left\langle d|\hat{H}| f_{\varepsilon}\right\rangle a_{f}(t, \varepsilon) \\
& =\mu_{\mathrm{ex}} \mathcal{E}(t) a_{i}(t)+E_{d} a_{d}(t)+\int d \varepsilon V^{*}(\varepsilon) a_{f}(t, \varepsilon) \\
i \dot{a}_{f}(t, \varepsilon) & =\left\langle f_{\varepsilon}|\hat{H}| i\right\rangle a_{i}(t)+\left\langle f_{\varepsilon}|\hat{H}| d\right\rangle a_{d}(t)+\int d \varepsilon^{\prime}\left\langle f_{\varepsilon}|\hat{H}| f_{\varepsilon^{\prime}}\right\rangle a_{f}\left(t, \varepsilon^{\prime}\right) \\
& =\mu_{\text {ion }}(\varepsilon) \mathcal{E}(t) a_{i}(t)+V(\varepsilon) a_{d}(t)+\left(E_{f}+\varepsilon\right) a_{f}(t, \varepsilon)
\end{aligned}
$$

$E_{i}, E_{d}, E_{f}+\varepsilon$ denote the energies of the initial state, decaying state, and final state (with the emitted electron), respectively. The light-matter interaction is described by the transition dipole moment interacting with the external field $\mathcal{E}(t)$, with $\mu_{\mathrm{ex}}$ and $\mu_{\mathrm{ion}}(\varepsilon)$ representing the transition dipole moment of the photoexcitation and the direct photoionization process, respectively. Assuming a linear polarized field in $z$ direction, the transition dipole moment can be evaluated using MCTDH according to

$$
\begin{gathered}
\mu_{\mathrm{ex}}=\langle d|\hat{z}| i\rangle=\int_{-\infty}^{\infty} d z z \Phi_{d}^{*}(z) \Phi_{i}(z), \\
\mu_{\mathrm{ion}}(\varepsilon)=\left\langle f_{\varepsilon}|\hat{z}| i\right\rangle=\int_{-\infty}^{\infty} d z z \Phi_{f}^{*}(z, \varepsilon) \Phi_{i}(z) .
\end{gathered}
$$


Finally, $V(\varepsilon)$ denotes the transition matrix element from state $|d\rangle$ to $\left|f_{\varepsilon}\right\rangle$ via Coulomb interaction and hence $V(\varepsilon)=\left\langle f_{\varepsilon}\left|\hat{V}_{\text {coul }}\right| d\right\rangle$. Note that the radiative decay from $|i\rangle$ to $|d\rangle$ is negligible, since it was found to be three orders of magnitude slower than all other relevant transitions [25].

The final state amplitudes $a_{f}(t, \varepsilon)$ in Eq. (30) can be formulated as,

$$
a_{f}(t, \varepsilon)=-i \int_{t_{0}}^{t} d t^{\prime}\left[\mu_{\mathrm{ion}}(\varepsilon) \mathcal{E}\left(t^{\prime}\right) a_{i}\left(t^{\prime}\right)+V(\varepsilon) a_{d}\left(t^{\prime}\right)\right] e^{i\left(E_{f}+\varepsilon\right)\left(t^{\prime}-t\right)} .
$$

We insert this representation of $a_{f}(t, \varepsilon)$ into the integral in Eq. (29). To solve the improper integral we apply the local approximation [72], which limits the energy of the free electron to a maximum value. This yields

$$
\int_{0}^{\infty} d \varepsilon V^{*}(\varepsilon) a_{f}(t, \varepsilon)=-\frac{i}{2} \gamma \mathcal{E}(t) a_{i}(t)-\frac{i}{2} \Gamma_{\mathrm{ICD}} a_{d}(t)
$$

with $\gamma=2 \pi V^{*} \mu_{\text {ion }}, \Gamma_{\text {ICD }}=2 \pi|V|^{2}$. Similarly, the integral in Eq. (28) now reads

$$
\int_{0}^{\infty} d \varepsilon \mu_{\mathrm{ion}}^{*} a_{f}(t, \varepsilon)=-\frac{i}{2} \Gamma_{\text {ion }}^{*} \mathcal{E}^{*}(t) a_{i}(t)-\frac{i}{2} \gamma^{*} a_{d}(t),
$$

with the field-independent $\Gamma_{\text {ion }}=2 \pi\left|\mu_{\text {ion }}\right|^{2}$.

Thus, the equations of motion used in the state-resolved calculation read

$$
\begin{aligned}
i \dot{a}_{i}(t) & =\left(E_{i}-\frac{i}{2} \Gamma_{\mathrm{ion}}^{*}|\mathcal{E}(t)|^{2}\right) a_{i}(t)+\left(\mu_{\mathrm{ex}}^{*}-\frac{i}{2} \gamma^{*}\right) \mathcal{E}^{*}(t) a_{d}(t), \\
i \dot{a}_{d}(t) & =\left(\mu_{\mathrm{ex}}-\frac{i}{2} \gamma\right) \mathcal{E}(t) a_{i}(t)+\left(E_{d}-\frac{i}{2} \Gamma_{\mathrm{ICD}}\right) a_{d}(t), \\
i \dot{a}_{f}(t, \varepsilon) & =\mu_{\mathrm{ion}} \mathcal{E}(t) a_{i}(t)+V a_{d}(t)+\left(E_{f}+\varepsilon\right) a_{f}(t, \varepsilon) .
\end{aligned}
$$

The occupations of the two-electron states are readily available via $P_{i}(t)=$ $\left|a_{i}(t)\right|^{2}, P_{d}(t)=\left|a_{d}(t)\right|^{2}, P_{f}(t)=\int d \varepsilon\left|a_{f}(t, \varepsilon)\right|^{2}$. From $P_{d}(t)$ the spectrum can be evaluated as described in Sec. 2.2.1. Eqs. (36)-(38) were solved 
numerically with a self-developed fortran program. Most of the required parameters were obtained from previous MCTDH calculations, namely $E_{i}=$ -0.9180 a.u. $(-9.4570 \mathrm{meV}), E_{d}=E_{f}+\varepsilon=-0.4157$ a.u. $(-4.2825 \mathrm{meV})$, $\omega=0.5023$ a.u. $(5.1746 \mathrm{meV}), t_{w}=258.5629$ a.u. $(16.5204 \mathrm{ps}), \Gamma_{\mathrm{ICD}}=$ $3.9679 \cdot 10^{-4}$ a.u. $\left(4.0876 \cdot 10^{-3} \mathrm{meV}\right.$, as a rate $\left.6.21 \cdot 10^{-3} 1 / \mathrm{ps}\right), \mu_{\mathrm{ex}}=0.8923$ a.u. $(9.1922 \mathrm{meV})$, and $\varepsilon_{0}=0.0243$ a.u., which corresponds to an intensity of $I=2.8918 \cdot 10^{8} \mathrm{~W} / \mathrm{cm}^{2}$. The determination of $\Gamma_{\text {ion }}$ and hence $\mu_{\text {ion }}$ will be motivated in Sec. 3.1.

\section{Results and Discussion}

\section{1. $\pi$-Pulse Induced ICD}

Before laying out the full spectral range of the resonant laser pulses we go back to the already investigated $\pi$-pulse excitation [51] that yields a single inversion from the ground into the resonant excited state. First of all we analyze the details of the excitation dynamics calculated with MCTDH, using the grid size and box length which had been optimized in a previous study, where it was shown not to perturb the ICD decay process [43].

The top and bottom panel of Fig. 2 show the projections of the total wavefunction on the one- and two-electron bound states from the MCTDH calculations as defined in Eqs. (18) and (22). On the one hand the state $\left|L_{0} R_{0}\right\rangle$ (see population shown in dark gray line, Fig. 2, bottom) is fully depopulated at the end of the pulse duration, namely at $16.5 \mathrm{ps}$, echoing the well-known population inversion induced by the $\pi$ pulse. Since this population never recovers from zero, processes such as radiative recombination and electron tunneling can be ruled out. On the other hand, the resonance state 


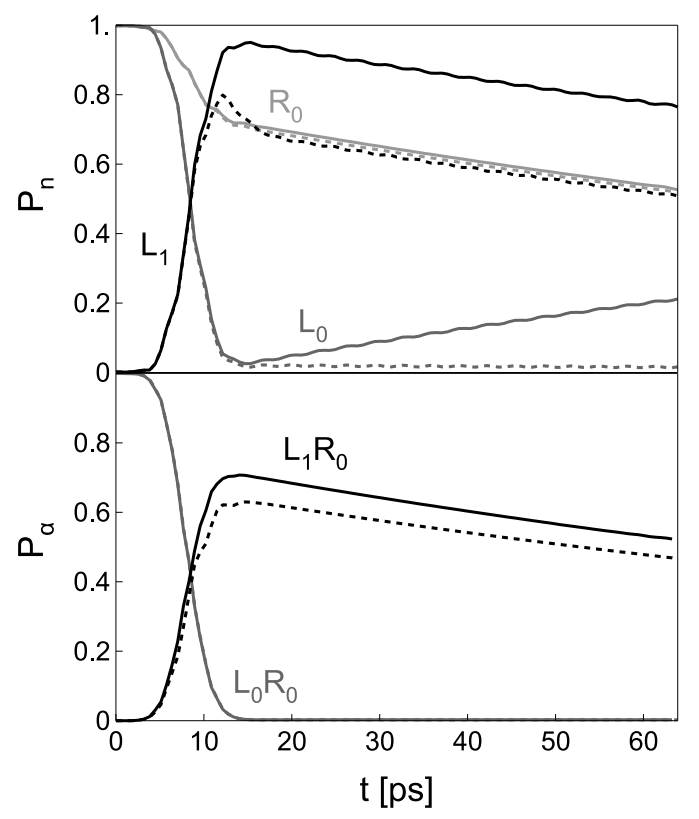

Figure 2: Populations of one-electron (upper panel) and two-electron bound states (lower panel) of the paired QD ICD process. The system is initialized by a $\pi$ pulse which lasts for 16.5 ps. Two different boundary conditions with distinct grid sizes are employed: Dashed lines correspond to MCTDH computations using a 140-point DVR grid with CAP, while solid lines correspond to calculations performed using a 980-point DVR grid without CAP.

$\left|L_{1} R_{0}\right\rangle$ (black line), populated via a photoexcitation while competing with the direct photoionization channel to $\left|L_{0} \varepsilon\right\rangle$, reaches its maximum population $P_{L_{1} R_{0}}^{\max }$ at $t=14.1 \mathrm{ps}$, i.e. prior the end of pulse duration, and decays exponentially afterwards.

The major information from Fig. 2 is the comparison of computations, using two different boundary conditions: one is a short grid interval $[-541.78$ $\mathrm{nm}, 541.78 \mathrm{~nm}$ ] with 140 sine DVR points and a complex absorbing potential (CAP, cf. Sec. 2.2.1), while the other is an identically-spaced long-grid interval [-3792.42 nm, $3792.42 \mathrm{~nm}]$ with 980 sine DVR points but without 
CAP. For each of the grids the resonant excitation energy for the pulse of Eq. (14) is deduced from relaxation calculations to be $\omega_{140}=5.1746 \mathrm{meV}$ and $\omega_{980}=5.1791 \mathrm{meV}$. The other laser parameters were determined from the Rabi oscillations under permanent irradiation. The values $t_{w, 140}=16.5204$ ps and $\varepsilon_{0,140}=0.0243004$ a.u. $\left(I_{140}=2.8918 \cdot 10^{8} \mathrm{~W} / \mathrm{cm}^{2}\right)$ as well as $t_{w, 980}=16.5062 \mathrm{ps}$ and $\varepsilon_{0,980}=0.0243213$ a.u. $\left(I_{980}=2.8943 \cdot 10^{8} \mathrm{~W} / \mathrm{cm}^{2}\right)$, respectively, were applied.

Once the pulse is turned off, the populations of $\left|L_{1} R_{0}\right\rangle$ obtained from both boundary conditions, decrease with a similar rate proving that the evaluated ICD decay width $\left(\Gamma_{\mathrm{ICD}}^{140}=4.0876 \cdot 10^{-3} \mathrm{meV}\right.$ and $\left.\Gamma_{\mathrm{ICD}}^{980}=4.1160 \cdot 10^{-3} \mathrm{meV}\right)$ indeed does not depend on the used boundary condition (up to a certain numerical accuracy, of course). However, employing a CAP in a short grid interval leads to a lower overall population of the $\left|L_{1} R_{0}\right\rangle$ state (Fig. 2, bottom, black line) as shown by the associated $P_{L_{1} R_{0}}$ (dashed line) compared to the one without CAP (solid line). Such effect was not reported in our previous calculations $[25,43]$, due to the fact that we were only interested in the decay of an initial state $\left|L_{1} R_{0}\right\rangle$, which turned out to be hardly influenced by the choice of the boundary condition. Hence, the effect must originate from the laser excitation into either $\left|L_{1} R_{0}\right\rangle$ or $\left|L_{0} \varepsilon\right\rangle$. Since the eigenfunction of the resonance excited state $\left|L_{1} R_{0}\right\rangle$ is quite localized, we expect the same population increase for both grids by the laser. Contrary, the $\left|L_{0} \varepsilon\right\rangle$ states that are populated as well directly via the pulse are as wide as the complete grid and hence reach into the CAP. In this sense the CAP may accelerate photoionization and lead to a smaller $P_{L_{1} R_{0}}^{\max }$.

Another difference of the two grids appears for the single-electron projec- 
tions. With the CAP-induced enhancement of direct ionization the $L_{1}$ level (upper panel of Fig. 2, black dashed line) is unphysically little populated after the pulse with a pronounced peak near the end of the pulse. Besides, employing the CAP with a short grid yields a constant population of the $L_{0}$ level (dark gray, dashed line) when the ICD process takes place, i.e. the $L_{1}$ level population decreases. In contrast, the population of $L_{0}$ obtained from the long grid calculation (black and dark gray solid lines) increases steadily counterbalancing the $P_{L 1}$ behavior. This is what one would expect from an ICD process, i.e. the electron on level $L_{1}$ relaxes back to the level $L_{0}$ while the excess energy is used to ionized the electron from $R_{0}$ (light gray lines). In an earlier work [51] the unphysical behavior of $P_{L_{0}}(t)$ was corrected via a renormalization of the wavefunction, namely $P_{L_{0}}^{\prime}(t)=P_{L_{0}}(t)+\left(1-|\Psi(t)|^{2}\right)$. This suggests that the electron absorbed by the CAP must have been in the $\left|L_{0} \varepsilon\right\rangle$ state. This means by implication that multi-photon processes where not taken into account in that renormalization. Only the populations of $R_{0}$ (light gray lines) do not show differences comparing the two grids.

Although the calculation with a long grid without CAP can describe more accurately the details of the dynamics, such an accurate dynamics lasts no more than 64 ps. After that, the outgoing electronic wavepacket encounters the end of grid and is scattered back to the paired QD. Therefore, if only the decay rate of the resonance state is of interest, using a short grid with CAP provides highly-accurate results, only that one will have an artificially larger direct ionization rate than using the long-grid calculation.

In Fig. 3 we compare the transient resonance-state probability $P_{L_{1} R_{0}}$ obtained from the space-resolved MCTDH calculation (dotted line) with that 


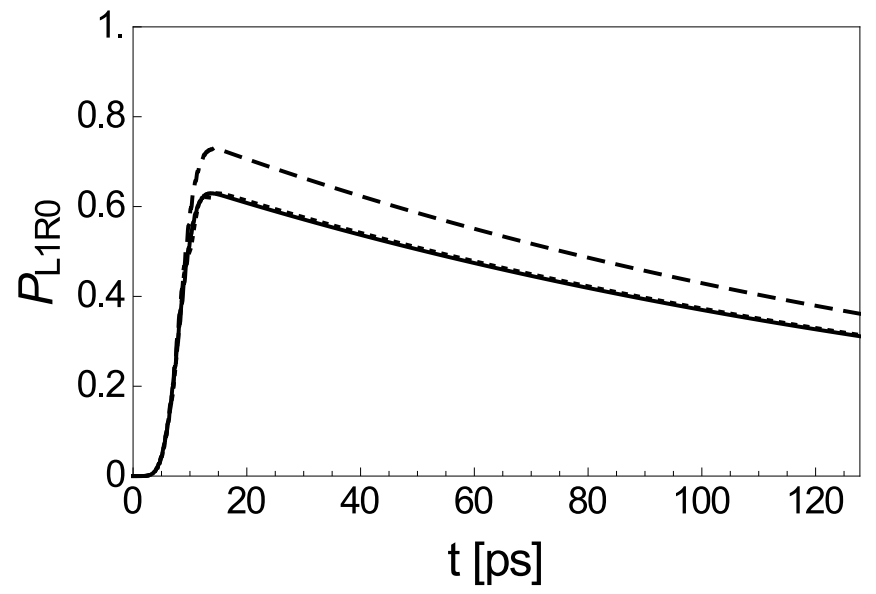

Figure 3: Projection of the total wavefunction on the $\left|L_{1} R_{0}\right\rangle$ resonance state for ICD initiated via a $16.5 \mathrm{ps} \pi$ pulse. The dotted line corresponds to the space-resolved MCTDH calculation on the small grid, the other lines to state-resolved calculations. As detailed in the text the solid curve is evaluated via an effective $\Gamma_{\mathrm{ion}, \varepsilon_{0}}^{\mathrm{fit}}=0.045 \mathrm{meV}$ obtained from an MCTDH calculation. The dashed curve is evaluated using $\Gamma_{\mathrm{ion}, \varepsilon_{0}}^{\mathrm{est}}=0.027 \mathrm{meV}$, which is obtained from the non-interacting two-electron system under a continuous lasing field.

obtained from the state-resolved representation (other lines). The latter are significantly faster, but require an a-priori calculation of all relevant energies and transition rates (cf. Sec. 2.2.2). Note that the ICD rate $\Gamma_{\mathrm{ICD}}=4.0876$. $10^{-3} \mathrm{meV}$ is straightforwardly obtained from MCTDH electron-dynamics calculations, whereas the calculation of the ionization rate $\Gamma_{\text {ion }}^{\mathrm{MCTDH}}$ is less intuitive. Evaluation of the transition matrix element $\Gamma_{\text {ion }}^{\mathrm{MCTDH}}=2 \pi\left|\left\langle f_{\varepsilon}|\hat{z}| i\right\rangle\right|^{2}$ is inaccurate when being done with the state functions obtained in the relaxation calculation, i.e. with a discretized continuum and a low integrationaccuracy on a non-dense grid. Moreover, $\left|f_{\varepsilon}\right\rangle$ contains only states accessible from single-photon excitations. Therefore, we prefer to estimate $\Gamma_{\text {ion }, \varepsilon_{0}}^{\text {est }}$ from a significantly more accurate MCTDH propagation as introduced in [51]. 
There, the non-interacting two-electron paired QD is exposed to a continuous laser so that $P_{L_{1} R_{0}}$ undergoes Rabi oscillations. The maxima of $P_{L_{1} R_{0}}$ decrease mainly due to ionization (including multi-photon ionization) and their connecting line shows an exponential decay with $\Gamma_{\text {ion }, \varepsilon_{0}}^{\text {est }}=2.7416 \cdot 10^{-2}$ $\mathrm{meV}$. Note that the rate estimated here is intrinsically dependent on the laser strengths and connects to the rate $\Gamma_{\text {ion }}$ used in the equations of Sec. 2.2.2 through $\Gamma_{\text {ion }}=\Gamma_{\text {ion, } n \varepsilon_{0}} /\left(n \varepsilon_{0} / 2\right)^{2}=2 \pi \mu_{\text {ion }}^{2}$. The state-resolved calculation with this rate gives a resonance state probability $P_{L_{1} R_{0}}(t)$ with the same trend as that from the space-resolved calculation but at higher overall probability (dashed line in Fig. 3). This overestimation can be assigned to the neglect of Coulomb interaction while evaluating $\Gamma_{\text {ion,n } \varepsilon_{0}}^{\text {est }}$, because the Coulomb repulsion should lead to an increase of the direct ionization probability. Hence, we propose to calibrate the state-resolved calculation by fitting its probability to the space-resolved probability for the known $\pi$-pulse scenario (solid line) before we transfer the method's usage to other pulses. This renders the larger $\Gamma_{\mathrm{ion}, \varepsilon_{0}}^{\mathrm{fit}}=4.5001 \cdot 10^{-2} \mathrm{meV}$. The other perspective for a more accurate state-resolved description of ionization would be to include the three additional sets of continuum states into the model that can be reached after a two-photon pulse. This would, however, complicate the algebra in the spirit of Sec. 2.2 .2 considerably, and require the evaluation of further transition matrix elements with the difficulties described in the above paragraph.

Let us now turn to the photoelectron spectrum (Fig. 4 (c)). It is widely known that an ionization spectrum has a typical profile when the ionization process into a specific final state can take place via two distinct pathways, 

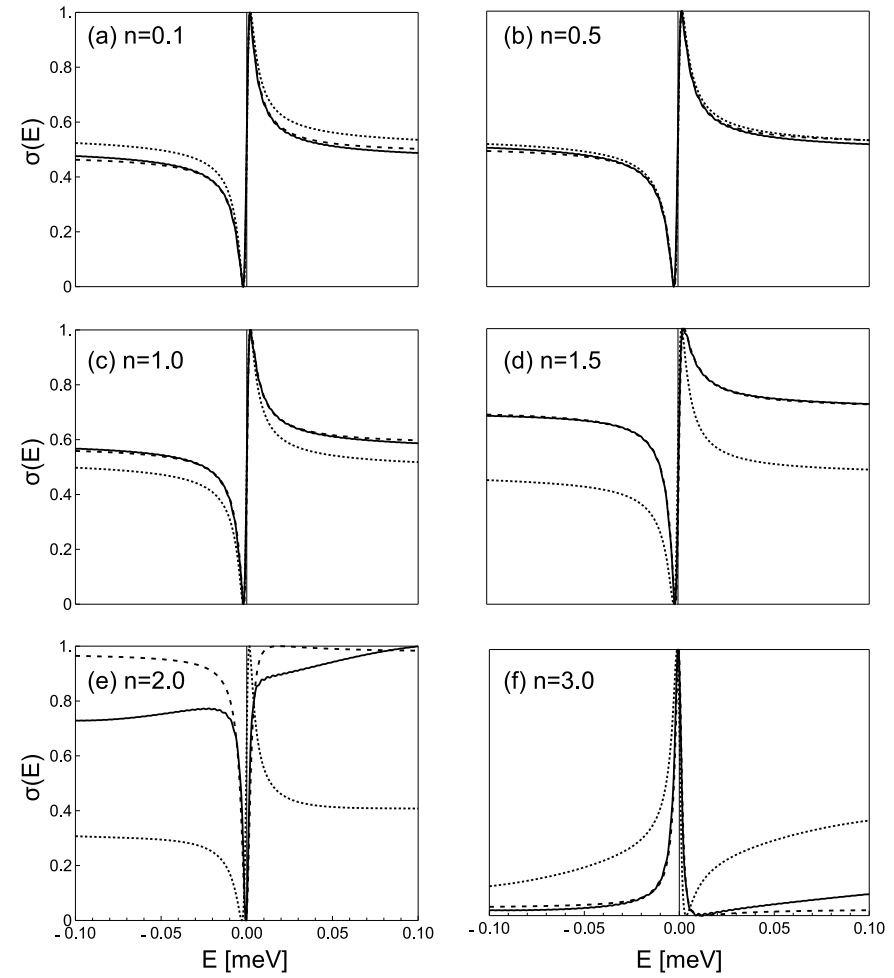

Figure 4: Series of Fano profiles for different $n$ predicted from the calculation of the electron spectra (Eq. (23)). Line shapes correspond to space-resolved calculations (solid), the fit of the profile these data (dashed) as well as state-resolved calculations (dotted), without showing the respective fit explicitly. For the smaller $n \varepsilon_{0}((\mathrm{a})-(\mathrm{d}))$ the Fano profiles are asymmetric along the ordinate. They become nearly ideal ((b),(c)) for the $0.5 \pi$ and $1 \pi$ pulse and asymmetric with respect to the abscissa for the largest $n \varepsilon_{0}((\mathrm{e}),(\mathrm{f}))$. 
i.e. via continuum states or via a discrete state [59, 60]. Depending on the interrelation of the lifetime of the resonance state and the applied external field, i.e. pulse strength and duration, the resulting profile can be peak multiplets $[58,53]$ or Fano peaks $[51,59,60,61]$ (cf. Sec. 1). As in our present study the decay of the resonance state is slower than the duration of a Rabi oscillation we observe a nearly-ideal Fano profile for an $n \pi$ pulse (Fig. 4 (c), solid line). In Ref. [51] we showed how the absolute ICD rate and the parameter $q$ can be deduced from the shape when fitting with Eq. (24) (dashed line). Here we find $\Gamma_{\mathrm{ICD}}=3.91 \cdot 10^{-3} \mathrm{meV}$ in reasonable agreement with $\Gamma_{\text {ICD }}$ obtained when fitting the exponential decay of $P_{L_{1} R_{0}} \cdot q=0.854$ further suggests an approximately equal importance of both pathways. The Fano profile obtained from the state-resolved ansatz (dotted line) gives the same $\Gamma_{\mathrm{ICD}}=3.95 \cdot 10^{-3} \mathrm{meV}$, however, a slightly higher $q=0.986$ (cf. Tab. 2) which we will discuss in later sections.

\subsection{ICD After Arbitrary Laser Pulses}

Another point of this paper is to investigate how an arbitrarily-strong pulsed laser can trigger ICD in competition with direct ionization. Shown by the black solid line in Fig. 5 is how the ionization probability $\left(1-\left|\Psi\left(t_{\text {end }}\right)\right|^{2}\right)$ varies with the laser intensity. The quantity $1-\left|\Psi\left(t_{e n d}\right)\right|^{2}$ evaluated after a long MCTDH propagation time $t_{\text {end }}=1277.86 \mathrm{ps}$ is an indicator for the total ionization probability of the system for either pathway, the direct one or ICD. For very weak fields $1-\left|\Psi\left(t_{\text {end }}\right)\right|^{2}=0$ means that no ICD or ionization has occurred. The system remained in the ground state and the norm $\left|\Psi\left(t_{\text {end }}\right)\right|^{2}=$ 1 was conserved. The contrary case occurs for the first time at $\varepsilon_{0}=0.0243$ a.u. ( $\pi$ pulse), where the system is either directly ionized or populates the 


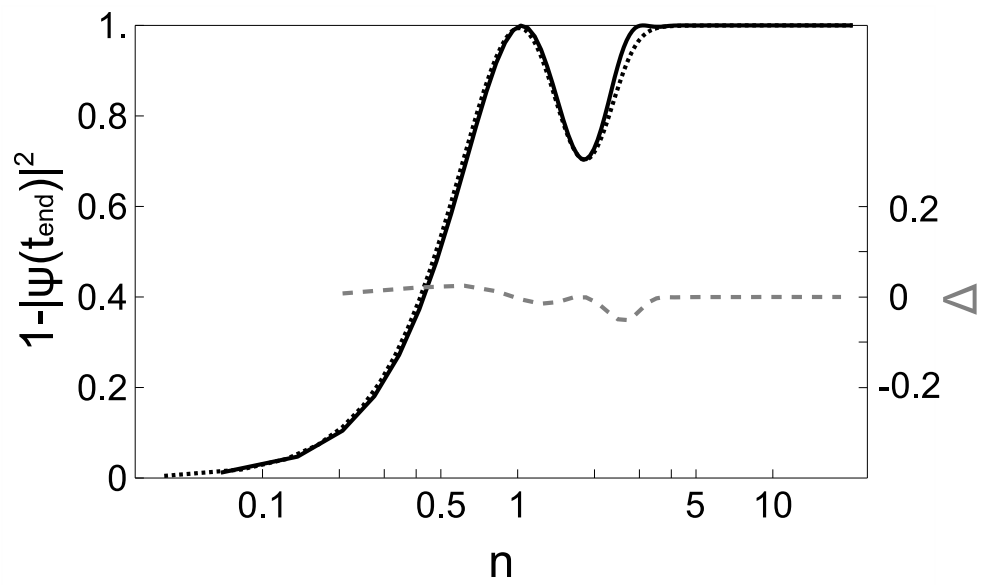

Figure 5: Scan of the ionization probability $1-\left|\Psi\left(t_{e n d}\right)\right|^{2}$ as function of $n$, a measure for the laser strength $\left(n \varepsilon_{0}\right)$, for the space-resolved (solid black line) and the state-resolved (black dotted line) calculations. The difference $\Delta$ between the two curves (shifted up by 0.4 ) is depicted by the dotted gray line.

eventually completely decaying $\left|L_{1} R_{0}\right\rangle$ through population inversion. The loss of the norm is here due to the usage of the CAPs for the space-resolved calculations, which removes the electrons from the continuum. For the stateresolved calculations the ionization probability corresponds to the occupation of the continuum state $\left|L_{0} \varepsilon\right\rangle$ at $t_{\text {end }}, P_{L_{0} \varepsilon}(t)=1-P_{L_{0} R_{0}}(t)-P_{L_{1} R_{0}}(t)$.

In the range from $\pi$ to $5 \pi$ the total ionization probability oscillates. In principle it reaches minima at all even- $n \pi$ pulses, as such pulses render the system in the ground state $\left|L_{0} R_{0}\right\rangle$ and hence allow ICD to take place only during the short lasing period $t<t_{\omega}$. At the same time, direct ionization can happen and further depopulates the state $\left|L_{0} R_{0}\right\rangle$. This leads to non-zero minima as shown in Fig. 5. The height of population, 0.7 (0.99) for the $2 \pi(4 \pi)$ pulse, is even a direct measure for the direct photoionization which gains importance towards higher $n$. The ionization probability reaches its 
maxima at $n$ associated with odd- $n \pi$ pulses, which render the system into $\left|L_{1} R_{0}\right\rangle$, which then can decay completely via ICD.

Fig. 5 contains two scans, the one from space-resolved (solid, black line) and the one from state-resolved (dotted, black line) calculations, where for the latter the fitted $\Gamma_{\mathrm{ion}, \varepsilon_{0}}^{\mathrm{fit}}$ was used. Both lines are in great accord with each other. Therefore we show the scaled difference plot $\Delta=\left|\Psi^{\text {space }}\left(t_{\text {end }}\right)\right|^{2}-$ $\left|\Psi^{\text {state }}\left(t_{\text {end }}\right)\right|^{2}+0.4$ (right coordinate axis, light gray, dashed line). The largest deviations is in the range $2<n<4$ where the state-resolved calculation gives lower $1-\left|\Psi\left(t_{\text {end }}\right)\right|^{2}$ and hence underestimates direct ionization, i.e. the total ionization probability lags behind. This difference may be assigned to the rising importance of multi-photon effects that are contained in $\Gamma_{\text {ion }, \varepsilon_{0}}^{\text {fit }}$ only to an amount relevant for lower intensity radiation. Further increasing the pulse strength results in a saturated ionization probability in both calculations.

For $n<1$ the state-resolved calculation gives a slightly larger $1-\left|\Psi\left(t_{\text {end }}\right)\right|^{2}$. This may indicate a larger account for direct ionization which was introduced when fitting $\Gamma_{\text {ion, } \varepsilon_{0}}^{\mathrm{fit}}$ for a higher-energy $\pi$ pulse. But as the difference is small, we conclude that there is no obvious multi-photon effect. This conclusion is supported by a direct comparison of the state populations, obtained from both, space-resolved and state-resolved, calculations (cf. Sec. 3.2.3).

\subsection{1. $n \pi$ Pulses}

After visiting a few marked spots of the scan and discussing their characteristics, we begin our discussion on ICD after the odd- $n \pi$ pulses with $n=3,5$, which lead our system to the excited resonance state, and the even- $n \pi$ pulses $n=2,4$ that drive the system $n / 2$ times into the excited state and each time back into the ground state, so that the final situation 


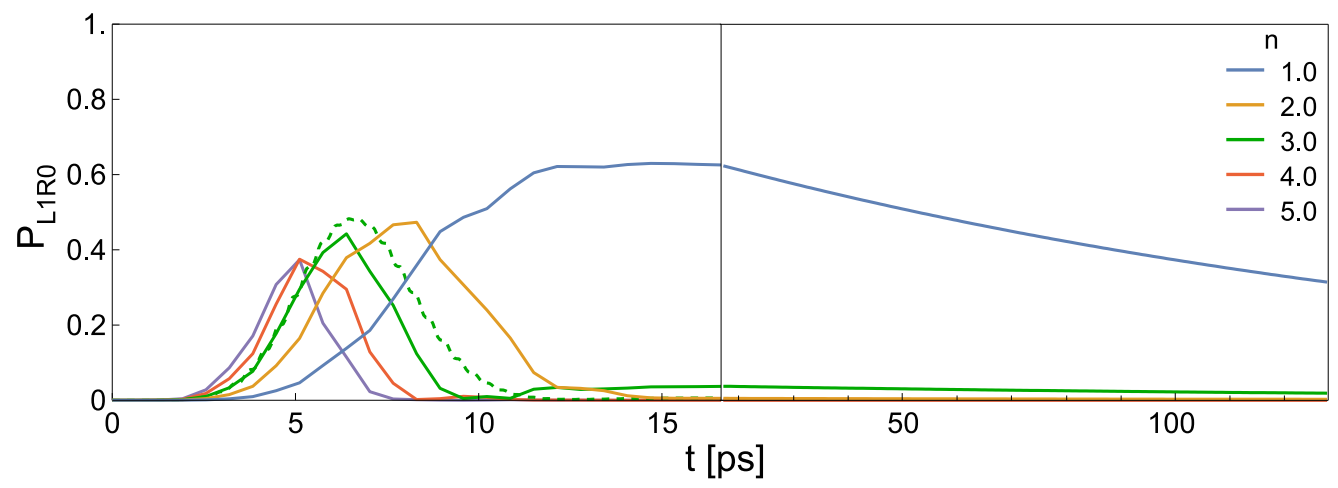

Figure 6: Population of the resonance state $\left|L_{1} R_{0}\right\rangle$ as it evolves through time under the radiation of $n \pi$ pulses. Note that the time scale is changed after $t>17 \mathrm{ps}$ to reveal the dynamics of the laser-induced Rabi oscillations and the ICD process, respectively. The space-resolved calculation (solid line) is compared to the state-resolved one (dashed line) for the case $n=3.0$.

is a non-excited system. In Fig. $6 P_{L_{1} R_{0}}$ obtained from the space-resolved calculations is shown for different puls strengths and Tab. 1 lists the data, such as $P_{L_{1} R_{0}}^{\max }$, obtained from these calculations. $P_{L_{1} R_{0}}$ for the case of the $3 \pi$ pulse is shown in a comparison for space-and state-resolved results (solid and dashed green line, respectively). In the state-resolved picture the maximum after a Rabi half cycle is at slightly higher $P_{L_{1} R_{0}}^{\max }$. This supports that its ionization probability is underestimated as already discussed in Sec. 3.1. For $t>11$ ps the state-resolved $P_{L_{1} R_{0}}$ is smaller than that of the space-resolved calculation.

At short times $t<17$ ps we use a higher resolution in Fig. 6 to visualize the number of Rabi oscillations. For longer times $17 \mathrm{ps} \leq t \leq 128$ ps we reduce the resolution of the time axis to show the exponential decay of $P_{L_{1} R_{0}}$ which is, however, only very clear in the $n=1$ case (blue line) from which 
$\Gamma_{\text {ICD }}$ was deduced. During the even- $n \pi$ pulse we see $n / 2$ maxima during the pulse, for $n=2$ at $t_{\max }=8 \mathrm{ps} \approx t_{\omega} / 2$ (yellow line) and for $n=4$ at $t_{\max }=5 \mathrm{ps} \approx t_{\omega} / 4$ and at $10 \mathrm{ps} \approx 3 t_{\omega} / 4$ (red line). In fulfillment of expectations after $n / 2$ full Rabi oscillations no ICD can be observed after the termination of the pulse as $P_{L_{1} R_{0}}$ is then nearly zero, hence $\Gamma_{\text {ICD }}$ remains unknown. But also after the odd- $n \pi$ pulses $P_{L_{1} R_{0}}$ is already below 0.1 and only for the $3 \pi$ pulse $\Gamma_{\mathrm{ICD}}=4.0879 \cdot 10^{-3} \mathrm{meV}$ can be obtained with a deviation below one per mille. An easier fitting would be obtained if direct ionization was less important as e.g. when the pulse was not applied to the right QD [51].

The evidence of the fact that increasing the intensity of laser field enhances the direct ionization process is observed from the simultaneously decreasing maximum populations $P_{L_{1} R_{0}}^{\max }$ (Tab. 1 and left panel of Fig. 6). Similarly, the recurrence of the successive maximum of $P_{L_{1} R_{0}}$ drops with the increased field strength. We also compare the space- with the state-resolved dynamics for higher $n$ and show it $n=3$ in Fig. 6 (dotted green line). For the $\pi$ pulse itself, where we had fitted $\Gamma_{\text {ion }}$ to match both functions (Sec. 3.1), both representations give identical results. But for larger $n P_{L_{1} R_{0}}^{\max }$ is typically larger at early times in the state-resolved representation. This effect has to be assigned to an underestimation of multi-photon ionization. In MCTDH multi-photon excitations are automatically included as all continuum states are included in the calculation and treated on equal footing. Contrary, in the state-resolved picture we do only consider single-photon excitation due to the underlying two-state plus continuum representation (Fig. 1). Any multi-photon effect could only be included through $\Gamma_{\text {ion, } \varepsilon_{0}}^{\text {fit }}$ which, however, 
Table 1: Maximal resonance state populations $P_{L_{1} R_{0}}^{\max }$ during the first Rabi cycle at $t_{\max }$ for $n \pi$ pulses with intensity $I$ read from Fig. 6 .

\begin{tabular}{|c|c|c|c|}
\hline$n$ & $I / \mathrm{Wcm}^{-2}$ & $P_{L_{1} R_{0}}^{\max }$ & $t_{\max } / \mathrm{ps}$ \\
\hline 1.0 & $2.8918 \cdot 10^{8}$ & 0.63 & 14.0 \\
\hline 2.0 & $5.7835 \cdot 10^{8}$ & 0.50 & 7.7 \\
\hline 3.0 & $8.6753 \cdot 10^{8}$ & 0.43 & 6.2 \\
\hline 4.0 & $1.1567 \cdot 10^{9}$ & 0.39 & 5.5 \\
\hline 5.0 & $1.4459 \cdot 10^{9}$ & 0.35 & 4.9 \\
\hline
\end{tabular}

was done for the $\pi$ pulse only.

As it turns out to be impossible to deduce a quantitative $\Gamma_{\text {ICD }}$ by fitting the exponential decay of $P_{L_{1} R_{0}}$ in Fig. 6, we consider the photoelectron spectra to gain this information. The respective spectra based on spaceresolved calculations for $n=1,2,3$ are to be found in Fig. 4 (c), (e), and (f) (solid lines) and those based on state-resolved calculations in the same figures (dotted lines). When fitting Fano lines shapes to the former according to Eq. (24) (dashed lines), we obtain $\Gamma_{\text {ICD }}^{\text {space }}$ and the Fano parameter $q^{\text {space }}$, as listed in Tab. 2. The respective values $q^{\text {state }}$ and $\Gamma_{\text {ICD }}^{\text {state }}$ are also given in Tab. 2. Note that it is easier to fit a reasonable $\Gamma_{\text {ICD }}$ to the profile connected to the $3 \pi$ pulse then to that of the $2 \pi$ pulse, because the former shows an exponentially decaying $P_{L 1 R 0}$. At this place we do not yet go into the details of $q$. We only want to state that $q$ for the $2 \pi$ pulse is in line with that for lower- $n \pi$ pulses (cf. Sec. 3.2.3), whereas that for the $3 \pi$ peaks out from the series. Here, we observe a large negative $q$. Such an effect was reported for a significant modification of the continuum by the laser $[73,74]$ and may eventually open 
Table 2: Fano profile parameters $q$ and $\Gamma_{\text {ICD }}$ from fits of the Fano profile (Eq. (24)) to photoelectron spectra of space- and state-resolved calculations (indicated by superscripts) for a series of $n \pi$ pulses with intensities $I$.

\begin{tabular}{|c|c|c|c|c|c|}
\hline$n$ & $I / \mathrm{Wcm}^{-2}$ & $q^{\text {space }}$ & $\Gamma_{\mathrm{ICD}}^{\text {space }} / \mathrm{meV}$ & $q^{\text {state }}$ & $\Gamma_{\mathrm{ICD}}^{\text {state }} / \mathrm{meV}$ \\
\hline 0.1 & $2.8918 \cdot 10^{7}$ & 1.035 & $3.99 \cdot 10^{-3}$ & 0.946 & $3.95 \cdot 10^{-3}$ \\
\hline 0.5 & $1.4459 \cdot 10^{8}$ & 0.976 & $3.92 \cdot 10^{-3}$ & 0.951 & $4.02 \cdot 10^{-3}$ \\
\hline 1.0 & $2.8918 \cdot 10^{8}$ & 0.854 & $3.91 \cdot 10^{-3}$ & 0.986 & $3.95 \cdot 10^{-3}$ \\
\hline 1.5 & $4.3376 \cdot 10^{8}$ & 0.644 & $4.08 \cdot 10^{-3}$ & 1.053 & $4.14 \cdot 10^{-3}$ \\
\hline 2.0 & $5.7835 \cdot 10^{8}$ & 0.160 & $6.08 \cdot 10^{-3}$ & 1.351 & $4.50 \cdot 10^{-3}$ \\
\hline 3.0 & $8.6753 \cdot 10^{8}$ & -6.090 & $3.99 \cdot 10^{-3}$ & -1.835 & $4.10 \cdot 10^{-3}$ \\
\hline
\end{tabular}

the door to resonance profile control in the context of electron dynamics in quantum dots.

\subsubsection{Strong Fields}

For all fields above the Fano regime, i.e. for $n>3$ the scan of Fig. 5 shows the full realization of final state $\left|L_{0} \varepsilon\right\rangle$ predominantly through direct ionization as discussed in Sec. 3.1. In Fig. 6, for example, the $5 \pi$ excited system is excited into the resonance state once during the first Rabi cycle. No further Rabi inversion brings the system into the resonance state $\left|L_{1} R_{0}\right\rangle$ as all $\left|L_{0} R_{0}\right\rangle$ population has been used up through direct ionization. Neither from Fig. 6 nor from the photoelectron spectra $\Gamma_{\text {ICD }}$ can be deduced. Only in the case if a less-ionizing field, e.g. a pulse only applied to the left QD might change this situation [51]. 


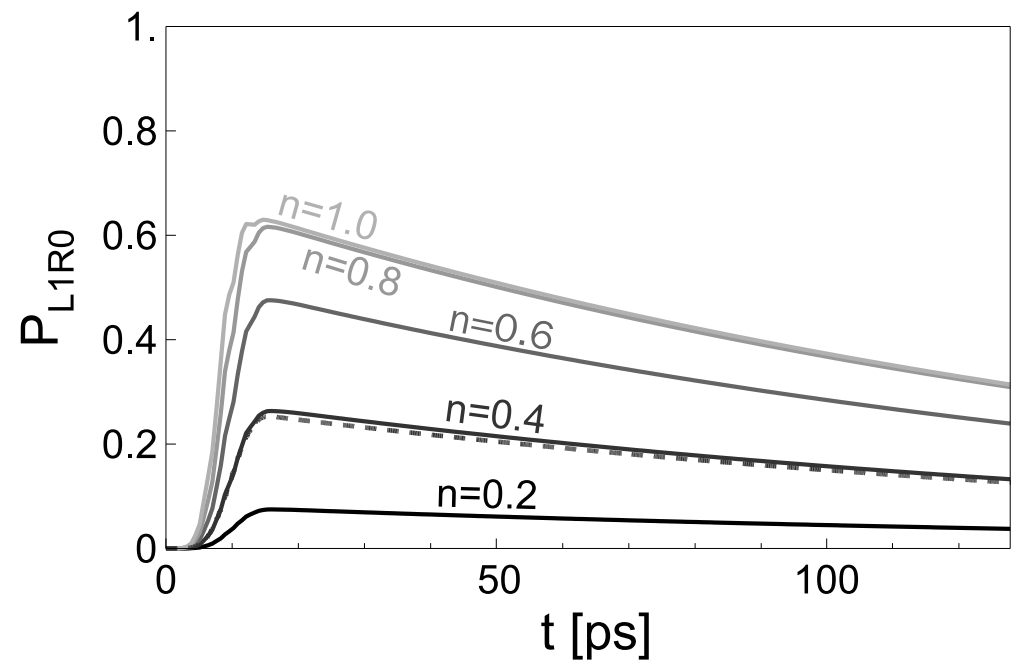

Figure 7: Population $P_{L_{1} R_{0}}$ of the resonance under weak laser pulses (below- $\pi$ ).

\subsubsection{Weak Fields}

The scan (Fig. 5) reveals a weak field regime of irrational- $n \pi$ laser pulses with $0<n<1$ in which only a partial population inversion is possible within the lasing duration $t_{w}$. Fig. 7 and Tab. 3 display the maxima of $P_{L_{1} R_{0}}$ at times $t_{\max }$ in dependence of $n \varepsilon_{0}$. After $t_{w} P_{L_{1} R_{0}}$ always decays exponentially and $\Gamma_{\text {ICD }}$ can be determined. A constant average $\left\langle\Gamma_{\text {ICD }}\right\rangle=4.0984 \cdot 10^{-3} \mathrm{meV}$ with small deviation is found for all pulses $n>0.2$. The deviation of the individual $\Gamma_{\text {ICD }}$ from that average increases with increasingly weak pulses over $2.34 \%$ for the $0.2 \pi$ pulse to $4.79 \%$ for the $0.1 \pi$ pulse. This is because the decay through ICD becomes faster than populating the decaying state via the laser pulse, i.e. $\partial P_{L_{1} R_{0}} / \partial t \approx 0$.

Let us briefly turn to the state-resolved calculations. In Fig. 7 the dotted line for $n=0.4$ represents the general trend found for all state-resolved curves. There $P_{L_{1} R_{0}}$ for the weaker fields is always slightly lower than that 
Table 3: Maximal resonance state populations $P_{L_{1} R_{0}}^{\max }$ at $t_{\max }$ before the exponential decay determined by $\Gamma_{\mathrm{ICD}}$ for $n \pi$ pulses with intensities $I$ obtained from Fig. 7 .

\begin{tabular}{|c|c|c|c|c|}
\hline$n$ & $I / \mathrm{Wcm}^{-2}$ & $P_{L_{1} R_{0}}^{\max }$ & $t_{\max } / \mathrm{ps}$ & $\Gamma_{\mathrm{ICD}} / \mathrm{meV}$ \\
\hline 0.1 & $2.8918 \cdot 10^{7}$ & 0.02 & 15.6 & $4.2836 \cdot 10^{-3}$ \\
\hline 0.2 & $5.7835 \cdot 10^{7}$ & 0.08 & 15.1 & $4.1831 \cdot 10^{-3}$ \\
\hline 0.4 & $1.1567 \cdot 10^{8}$ & 0.26 & 15.7 & $4.1159 \cdot 10^{-3}$ \\
\hline 0.6 & $1.7351 \cdot 10^{8}$ & 0.48 & 15.3 & $4.0976 \cdot 10^{-3}$ \\
\hline 0.8 & $2.3134 \cdot 10^{8}$ & 0.61 & 14.8 & $4.0922 \cdot 10^{-3}$ \\
\hline 1.0 & $2.8918 \cdot 10^{8}$ & 0.64 & 14.1 & $4.0876 \cdot 10^{-3}$ \\
\hline
\end{tabular}

from space-resolved calculations. Again this can be explained by the dynamics for multi-photon excitations. For $n<1$ these are over-represented in the state-resolved equations and hence the system is more strongly ionized.

In the discussed range the electron spectra can as well be analyzed. Fig. 4 (a)-(d) comprise a few relevant Fano shapes for different values of $n$, Tab. 2 the respective numerical data. In accord with the Fano theory of resonances [59] the profiles vary with increasing laser field strengths $n \varepsilon_{0}$ and intensities, respective, from a Lorentzian shape known for the pure decay $\left(q^{\text {space }} \rightarrow\right.$ $\infty, n=0$ [51]) to a quasi-ideal Fano profile for equal importance of both pathways $\left(q^{\text {space }}=0.854, n=1\right)$. This is also valid in the state-resolved picture. For intermediate $n$ the $q^{\text {space }}$ obtained in the MCTDH calculation decrease from 1.035 to 0.160 with increasing $n$, whereas the state-resolved approach gives an increase of $q^{\text {state }}$ from 0.946 to 1.351 for $0.1 \pi$ to $2.0 \pi$ pulses.

This brings us to the concrete meaning of the Fano profile parameter $q$. Fano himself defined $q$ as being proportional to the rate among the transi- 
tion probability from the initial into the resonance state with admixture of continuum contributions and that from the initial into the unperturbed continuum states [59]. As in MCTDH no unperturbed continuum is available, and on the other hand in the state-resolved calculations a resonance state with continuum admixture is not considered, the discussion in this paper can only be qualitative. For the space-resolved ansatz the denominator of the $q$ definition may eventually be more affected as the resonance state in MCTDH can adjust for varying continuum admixture. Upon increasing $n \varepsilon_{0}$ the transition into the continuum becomes more important, especially that into the multi-photon states which are explicitly included in MCTDH. Hence the denominator increases while $q^{\text {space }}$ decreases. Our initial understanding of $q=\% \mathrm{ICD} / \%$ ion [51] is in accord with this interpretation. In the stateresolved ansatz the denominator supposedly has a decreasing value, because the transition into always the same unperturbed continuum states is overestimated for small $n \varepsilon_{0}$ with the underlying constant $\Gamma_{\mathrm{ion}, \varepsilon_{0}}^{\mathrm{fit}}$, and in the inverse case underestimated. Hence, $q^{\text {state }}$ must increase here.

\section{Conclusions}

Nowadays the interatomic Coulombic decay is recognized as a highlyefficient elementary process in numerous physical and chemical systems [3, 4]. Ideas of its device application advanced to pairs of quantum wells [26] and quantum dots $[24,25]$ that offer a number of control options for ICD. Among these are differently-shaped, focused, and polarized initiating laser pulses [51].

This study targeted laser-intensity control. The present analyses of $n \pi$ 
pulses led to transient state populations, rates for ICD and direct ionization of the quantum dots, and probabilities for either pathway from photoelectron spectra. Particularly, we discussed the reason for Fano peak profiles dominating the spectrum and, additionally, the relevance of multi-photon excitations. The findings immediately revealed a below- $\pi$ to $\pi$ pulse for the most efficient preparation of the decaying state. In this spirit coherent control of ICD is within reach.

For the past and present space-resolved real-time dynamics predictions of ICD $[25,51]$ an antisymmetrized variant of the multi-configuration timedependent Hartree method has been used. Now we consider a state-resolved ansatz that was originally developed for the description of the resonant Auger decay in atoms $[70,71]$. From this complementary viewing angle we consolidate our recent and previous results.

\section{Acknowledgements}

When A.B. came to Heidelberg, L.C. was piecing together a puzzle: in the foreground two spheroids and flickering energy in between. The puzzle seemed half-way done, but miraculously more and more pieces materialized. L.C. explained the rules and was then eager to share and even hand over some pieces to A.B. She started putting them together with additional pieces into an independent side-motive and awaits the visionary picture when reconnecting it to the expanding puzzle. A.B. is deeply thankful for the opportunities that arose from L.C.'s complaisant support and congratulates him on his amazing achievements. Y.-C. C. thanks L.C. for his guidance during her $\mathrm{PhD}$ study, and congratulates him on his wonderful life as a scientist. 
The authors would also like to acknowledge discussion with Simona Scheit. This work was started while Y.-C. C. and A.B. were still employed at the University of Heidelberg. A.B. acknowledges financial support by the Volkswagen Foundation (Freigeist fellowship no. 89525) and A.H. and A.B. by the Deutsche Forschungsgemeinschaft (BA 3770).

[1] K. Sakai, S. Stoychev, T. Ouchi, I. Higuchi, M. Schöffler, T. Mazza, H. Fukuzawa, K. Nagaya, M. Yao, Y. Tamenori, A. I. Kuleff, N. Saito, K. Ueda, Electron-transfer-mediated decay and interatomic Coulombic decay from the triply ionized states in argon dimers, Phys. Rev. Lett. 106 (2011) 033401. doi:http://dx.doi.org/10.1103/PhysRevLett.106.033401.

[2] L. S. Cederbaum, J. Zobeley, F. Tarantelli, Giant Intermolecular Decay and Fragmentation of Clusters, Phys. Rev. Lett. 79 (24) (1997) 4778. doi:http://dx.doi.org/10.1103/PhysRevLett.79.4778.

[3] U. Hergenhahn, Interatomic and Intermolecular Coulombic Decay: The Early Years, J. Electron Spectrosc. Relat. Phenom. 184 (2011) 78. doi:http://dx.doi.org/10.1016/j.elspec.2010.12.020.

[4] T. Jahnke, Interatomic and intermolecular Coulombic decay: the coming of age story, J. Phys. B: At. Mol. Opt. Phys. 48 (2015) 082001. doi:http://dx.doi.org/10.1088/0953-4075/48/8/082001.

[5] K. Kreidi, T. Jahnke, T. Weber, T. Havermeier, X. Liu, Y. Morishita, S. Schössler, L. Schmidt, M. Schöffler, M. Odenweller, N. Neumann, L. Foucar, J. Titze, B. Ulrich, F. Sturm, C. Stuck, R. Wallauer, S. Voss, I. Lauter, H. K. Kim, M. Rudloff, H. Fukuzawa, G. Prümper, N. Saito, 
K. Ueda, A. Czasch, O. Jagutzki, H. Schmidt-Böcking, S. Stoychev, P. V. Demekhin, R. Dörner, Relaxation processes following 1s photoionization and Auger decay in $\mathrm{Ne}_{2}$, Phys. Rev. A 78 (2008) 043422. doi:http://dx.doi.org/10.1103/PhysRevA.78.043422.

[6] P. Kolorenč, N. Sisourat, Interatomic Coulombic decay widths of helium trimer: Ab initio calculations, J. Chem. Phys. 143 (2015) 224310. doi:http://dx.doi.org/10.1063/1.4936897.

[7] E. Fasshauer, Non-nearest neighbour ICD in clusters, New J. Phys. 18 (2016) 043028. doi:http://dx.doi.org/10.1088/1367-2630/18/4/043028.

[8] X. Ren, E. J. Al Maalouf, A. Dorn, S. Denifl, Direct evidence of two interatomic relaxation mechanisms in argon dimers ionized by electron impact, Nat. Commun. 7 (2016) 11093. doi:http://dx.doi.org/10.1038/ncomms11093.

[9] V. Averbukh, L. S. Cederbaum, Interatomic Electronic Decay in Endohedral Fullerenes, Phys. Rev. Lett. 96 (2006) 053401. doi:http://dx.doi.org/10.1103/PhysRevLett.96.053401.

[10] R. De, M. Magrakvelidze, M. E. Madjet, S. T. Manson, H. S. Chakraborty, First prediction of inter-Coulombic decay of C60 inner vacancies through the continuum of confined atoms, J. Phys. B: At. Mol. Opt. Phys. 49 (2016) 11LT01. doi:http://dx.doi.org/10.1088/09534075/49/11/11LT01.

[11] M. Magrakvelidze, R. De, M. H. Javani, M. E. Madjet, S. T. Manson, H. S. Chakraborty, Coherence of Auger and inter-Coulombic decay pro- 
cesses in the photoionization of Ar@C60 versus Kr@C60, Eur. Phys. J. D. 70 (2016) 96. doi:http://dx.doi.org/10.1140/epjd/e2016-60703-y.

[12] E. F. Aziz, N. Ottosson, M. Faubel, I. V. Hertel, B. Winter, Interaction between liquid water and hydroxide revealed by core-hole de-excitation, Nature 455 (2008) 89. doi:http://dx.doi.org/10.1038/nature07252.

[13] T. Jahnke, H. Sann, T. Havermeier, K. Kreidi, C. Stuck, M. Meckel, M. Schöffler, N. Neumann, R. Wallauer, S. Voss, A. Czasch, O. Jagutzki, A. Malakzadeh, F. Afaneh, T. Weber, H. Schmidt-Böcking, R. Dörner, Ultrafast energy transfer between water molecules, Nat. Phys. 6 (2010) 139. doi:http://dx.doi.org/10.1038/nphys1498.

[14] C. P. Schwartz, S. Fatehi, R. J. Saykally, D. Prendergast, Importance of Electronic Relaxation for Inter-Coulombic Decay in Aqueous Systems, Phys. Rev. Lett. 105 (2010) 198102. doi:http://dx.doi.org/10.1103/PhysRevLett.105.198102.

[15] S. Thürmer, M. Ončák, N. Ottosson, R. Seidel, U. Hergenhahn, S. E. Bradforth, P. Slavíček, B. Winter, On the nature and origin of dicationic, charge-separated species formed in liquid water on X-ray irradiation, Nat. Chem. 5 (2013) 590. doi:http://dx.doi.org/10.1038/nchem.1680.

[16] I. Unger, D. Hollas, R. Seidel, S. Thürmer, E. F. Aziz, P. Slavíček, B. Winter, Control of X-ray Induced Electron and Nuclear Dynamics in Ammonia and Glycine Aqueous Solution via Hydrogen Bonding, J. Phys. Chem. B 119 (2015) 10750. doi:http://dx.doi.org/10.1021/acs.jpcb.5b07283. 
[17] P. Slavíček, N. V. Kryzhevoi, E. F. Aziz, B. Winter, Relaxation Processes in Aqueous Systems upon X-ray Ionization: Entanglement of Electronic and Nuclear Dynamics, J. Phys. Chem. Lett. 7 (2016) 234. doi:http://dx.doi.org/10.1021/acs.jpclett.5b02665.

[18] S. D. Stoychev, A. I. Kuleff, L. S. Cederbaum, Intermolecular Coulombic decay in small biochemically relevant hydrogen-bonded systems, J. Am. Chem. Soc. 133 (2011) 6817. doi:10.1021/ja200963y.

[19] P. H. P. Harbach, M. Schneider, S. Faraji, A. Dreuw, Intermolecular Coulombic Decay in Biology: The Initial Electron Detachment from FADH- in DNA Photolyases, J. Phys. Chem. Lett. 4 (2013) 943. doi:http://dx.doi.org/10.1021/jz400104h.

[20] A. Dreuw, S. Faraji, A quantum chemical perspective on (6-4) photolesion repair by photolyases, Phys. Chem. Chem. Phys. 15 (2013) 19957. doi:http://dx.doi.org/10.1039/C3CP53313A.

[21] K. Gokhberg, P. Kolorenč, A. I. Kuleff, L. S. Cederbaum, Site- and energy-selective slow-electron production through intermolecular Coulombic decay, Nature $505 \quad$ (2014) 661. doi:http://dx.doi.org/10.1038/nature12936.

[22] E. Alizadeh, T. M. Orlando, L. Sanche, Biomolecular Damage Induced by Ionizing Radiation: The Direct and Indirect Effects of LowEnergy Electrons on DNA, Annu. Rev. Phys. Chem. 66 (2015) 379. doi:http://dx.doi.org/10.1146/annurev-physchem-040513-103605. 
[23] V. Stumpf, K. Gokhberg, L. S. Cederbaum, The role of metal ions in X-ray-induced photochemistry, Nat. Chem. 8 (2016) 237. doi:http://dx.doi.org/10.1038/nchem.2429.

[24] I. Cherkes, N. Moiseyev, Electron relaxation in quantum dots by the interatomic Coulombic decay mechanism, Phys. Rev. B 83 (2011) 113303. doi:http://dx.doi.org/10.1103/PhysRevB.83.113303.

[25] A. Bande, K. Gokhberg, L. S. Cederbaum, Dynamics of interatomic Coulombic decay in quantum dots, J. Chem. Phys. 135 (2011) 144112. doi:http://dx.doi.org/10.1063/1.3646205.

[26] T. Goldzak, L. Gantz, I. Gilary, G. Bahir, N. Moiseyev, Interatomic Coulombic decay in two coupled quantum wells, Phys. Rev. B 91 (2015) 165312. doi:10.1103/PhysRevB.91.165312.

[27] R. Santra, L. S. Cederbaum, Non-Hermitian electronic theory and applications to clusters, Phys. Rep. 368 (2002) 1. doi:http://dx.doi.org/10.1016/S0370-1573(02)00143-6.

[28] S. Barth, S. Joshi, S. Marburger, V. Ulrich, A. Lindblad, G. Öhrwall, O. Björneholm, U. Hergenhahn, Observation of resonant Interatomic Coulombic Decay in Ne clusters, J. Chem. Phys. 122 (2005) 241102. doi:http://dx.doi.org/10.1063/1.1937395.

[29] T. Aoto, K. Ito, Y. Hikosaka, E. Shigemasa, F. Penent, P. Lablanquie, Properties of resonant interatomic Coulombic decay in Ne dimers, Phys. Rev. Lett. 97 (2006) 243401. doi:10.1103/PhysRevLett.97.243401. 
[30] K. Gokhberg, A. B. Trofimov, T. Sommerfeld, L. S. Cederbaum, Ionization of metal atoms following valence-excitation of neighbouring molecules, Europhys. Lett. $72 \quad$ (2005) 228. doi:http://dx.doi.org/10.1209/epl/i2005-10227-7.

[31] K. Gokhberg, V. Averbukh, L. S. Cederbaum, Interatomic decay of inner-valence-excited states in clusters, J. Chem. Phys. 124 (2006) 144315. doi:http://dx.doi.org/10.1063/1.2187472.

[32] K. Gokhberg, L. S. Cederbaum, Environment assisted electron capture, J. Phys. B: At. Mol. Opt. Phys. 42 (2009) 231001. doi:http://dx.doi.org/10.1088/0953-4075/42/23/231001.

[33] K. Gokhberg, L. S. Cederbaum, Interatomic Coulombic electron capture, Phys. Rev. A. $82 \quad$ (2010) 052707. doi:http://dx.doi.org/10.1103/PhysRevA.82.052707.

[34] F. M. Pont, A. Bande, L. S. Cederbaum, Controlled energy-selected electron capture and release in double quantum dots, Phys. Rev. B 88 (2013) 241304(R). doi:http://dx.doi.org/10.1103/PhysRevB.88.241304.

[35] A. Bande, F. M. Pont, K. Gokhberg, L. S. Cederbaum, Interatomic Coulombic electron capture in atomic, molecular, and quantum dot systems, EPJ Web Conf. 84 (2015) 07002. doi:10.1051/epjconf/20158407002.

[36] F. M. Pont, A. Bande, L. S. Cederbaum, Electron-correlation driven capture and release in double quantum dots, J. Phys.: Cond. Matter. 28 (2016) 075301. doi:http://dx.doi.org/10.1088/0953-8984/28/7/075301. 
[37] H.-K. Kim, H. Gassert, M. S. Schöffler, J. N. Titze, M. Waitz, J. Voigtsberger, F. Trinter, J. Becht, A. Kalinin, N. Neumann, C. Zhou, L. P. H. Schmidt, O. Jagutzki, A. Czasch, H. Merabet, H. Schmidt-Böcking, T. Jahnke, A. Cassimi, R. Dörner, Ion-impact-induced interatomic Coulombic decay in neon and argon dimers, Phys. Rev. A 88 (2013) 042707. doi:10.1103/PhysRevA.88.042707.

[38] A. Bande, F. M. Pont, P. Dolbundalchok, K. Gokhberg, L. S. Cederbaum, Dynamics of interatomic Coulombic decay in quantum dots: Singlet initial state, EPJ Web Conf. 41 (2013) 04031. doi:http://dx.doi.org/10.1051/epjconf/20134104031.

[39] B. Marquardt, M. Geller, B. Baxevanis, D. Pfannkuche, A. D. Wieck, D. Reuter, A. Lorke, Probing excitons and biexcitons in coupled quantum dots by coherent two-dimensional optical spectroscopy, Nat. Commun. 2 (2011) 209. doi:http://dx.doi.org/10.1103/PhysRevB.79.155324.

[40] J. Salfi, S. Roddaro, D. Ercolani, L. Sorba, I. Savelyev, M. Blumin, H. E. Ruda, F. Beltram, Electronic properties of quantum dot systems realized in semiconductor nanowires, Semicond. Sci. Technol. 25 (2010) 024007. doi:http://dx.doi.org/10.1088/0268-1242/25/2/024007.

[41] T. Kodera and W. G. van der Wiel and K. Ono and S. Sasaki and T. Fujisawa and S. Tarucha, High-frequency manipulation of fewelectron double quantum dotstoward spin qubits, Phys. E 22 (2004) 518. doi:http://dx.doi.org/10.1016/j.physe.2003.12.059.

[42] W. G. van der Wiel, S. De Franceschi, J. M. Elzerman, 
T. Fujisawa, S. Tarucha, L. P. Kouwenhoven, Electron Transport through double quantum dots, Rev. Mod. Phys. 75 (2002) 1. doi:http://dx.doi.org/10.1103/RevModPhys.75.1.

[43] P. Dolbundalchok, A. Bande, Geometrical control of the interatomic Coulombic decay process in quantum dots for infrared photodetectors, J. Comput. Chem., DOI: 10.1002/jcc.24410. doi:http://dx.doi.org/10.1002/jcc.24410.

[44] F. Weber, E. F. Aziz, A. Bande, Tuning of ICD-emitter properties in charged semiconductor double quantum dot systems, unpublished results.

[45] V. Averbukh, P. Kolorenč, K. Gokhberg, S. Cederbaum, Quantum Chemical Approach to Interatomic Decay Rates in Clusters, Vol. 20 of Advances in the Theory of Atomic and Molecular Systems, Springer, Netherlands, 2009, p. 155. doi:http://dx.doi.org/10.1007/978-90-4812985-0_8.

[46] K. Kreidi, P. V. Demekhin, T. Jahnke, T. Weber, T. Havermeier, X. Liu, Y. Morishita, S. Schössler, L. Schmidt, M. Schöffler, M. Odenweller, N. Neumann, L. Foucar, J. Titze, B. Ulrich, F. Sturm, C. Stuck, R. Wallauer, S. Voss, I. Lauter, H. K. Kim, M. Rudloff, H. Fukuzawa, G. Prümper, N. Saito, K. Ueda, A. Czasch, O. Jagutzki, H. Schmidt-Böcking, S. Scheit, L. S. Cederbaum, R. Dörner, Photo- and Auger-Electron Recoil Induced Dynamics of Interatomic Coulombic Decay, Phys. Rev. Lett. 103 (2009) 033001. doi:http://dx.doi.org/10.1103/PhysRevLett.103.033001. 
[47] N. Sisourat, N. V. Kryzhevoi, P. Kolorenč, S. Scheit, L. S. Cederbaum, Impact of nuclear dynamics on interatomic Coulombic decay in a he dimer, Phys. Rev. A 82 (2010) 053401. doi:10.1103/PhysRevA.82.053401.

[48] N. Sisourat, N. V. Kryzhevoi, P. Kolorenč, S. Scheit, T. Jahnke, L. S. Cederbaum, Ultralong-range energy transfer by interatomic Coulombic decay in an extreme quantum system, Nat. Phys. 6 (2010) 508. doi:10.1038/nphys1685.

[49] Y.-C. Chiang, F. Otto, H.-D. Meyer, L. S. Cederbaum, Interrelation between the distributions of kinetic energy release and emitted electron energy following the decay of electronic states, Phys. Rev. Lett. 107 (2011) 173001. doi:http://dx.doi.org/10.1103/PhysRevLett.107.173001.

[50] A. I. Kuleff, L. S. Cederbaum, Tracing Ultrafast Interatomic Electronic Decay Processes in Real Time and Space, Phys. Rev. Lett. 98 (2007) 083201. doi:http://dx.doi.org/10.1103/PhysRevLett.98.083201.

[51] A. Bande, Dynamics of interatomic Coulombic decay in quantum dots induced by a laser field, J. Chem. Phys. 138 (2013) 214104. doi:http://dx.doi.org/10.1063/1.4807611.

[52] Y. Sajeev, N. Moiseyev, Theory of autoionization in twoelectron spherical quantum dots, Phys. Rev. B 78 (2008) 075316. doi:http://dx.doi.org/10.1103/PhysRevB.78.075316.

[53] N. Rohringer, R. Santra, Resonant Auger effect at high x-ray intensity, Phys. Rev. A 77 (2008) 053404. doi:10.1103/PhysRevA.77.053404. 
[54] H. Fattahi, H. G. Barros, M. Gorjan, T. Nubbemeyer, B. Alsaif, C. Y. Teisset, M. Schultze, S. Prinz, M. Haefner, M. Ueffing, A. Alismail, L. Vámos, A. Schwarz, O. Pronin, J. Brons, X. Tao Geng, G. Arisholm, M. Ciappina, V. S. Yakovlev, D.-E. Kim, A. M. Azzeer, N. Karpowicz, D. Sutter, Z. Major, T. Metzger, F. Krausz, Third-generation femtosecond technology, Optica 1 (2014) 45. doi:10.1364/OPTICA.1.000045.

[55] B. Winter, Liquid microjet for photoelectron spectroscopy, Nucl. Instr. Meth. Phys. Res. A 601 (2009) 139. doi:http://dx.doi.org/10.1016/j.nima.2008.12.108.

[56] H. Kamada, H. Gotoh, J. Temmyo, T. Takagahara, H. Ando, Exciton rabi oscillation in a single quantum dot, Phys. Rev. Lett. 87 (2001) 246401. doi:10.1103/PhysRevLett.87.246401.

URL http://link.aps.org/doi/10.1103/PhysRevLett.87.246401

[57] T. H. Stievater, X. Li, D. G. Steel, D. Gammon, D. S. Katzer, D. Park, C. Piermarocchi, L. J. Sham, Rabi oscillations of excitons in single quantum dots, Phys. Rev. Lett. 87 (2001) 133603. doi:10.1103/PhysRevLett.87.133603.

[58] K. Rzążewski, J. Zakrzewski, M. Lewenstein, J. W. Haus, Strong-field autoionization by smooth laser pulses, Phys. Rev. A 31 (1985) 2995. doi:10.1103/PhysRevA.31.2995.

[59] U. Fano, Effects of configuration interaction on intensities and phase shifts, Phys. Rev. 124 (1961) 1866. doi:http://dx.doi.org/10.1103/PhysRev.124.1866. 
[60] A. E. Miroshnichenko, S. Flach, Y. S. Kivshar, Fano resonances in nanoscale structures, Rev. Mod. Phys. 82 (2010) 2257. doi:10.1103/RevModPhys.82.2257.

URL http://link.aps.org/doi/10.1103/RevModPhys.82.2257

[61] C. Ott, A. Kaldun, P. Raith, K. Meyer, M. Laux, J. Evers, C. H. Keitel, C. H. Greene, T. Pfeifer, Lorentz meets Fano in spectral line shapes: A universal phase and its laser control, Science 340 (2013) 716. arXiv:http://science.sciencemag.org/content/340/6133/716.full.pdf, doi:10.1126/science.1234407.

[62] Electronic archive: New Semiconductor Materials. Characteristics and Properties, http://www.ioffe.ru/SVA/NSM/Semicond/GaAs/basic.html (accessed April 15, 2016). .

[63] I. I. Rabi, Space Quantization in a Gyrating Magnetic Field, Phys. Rev. 51 (1937) 652. doi:http://dx.doi.org/10.1103/PhysRev.51.652.

[64] H.-D. Meyer, U. Manthe, L. S. Cederbaum, The multi-configurational time-dependent Hartree approach, Chem. Phys. Lett. 165 (1990) 73. doi:http://dx.doi.org/10.1016/0009-2614(90)87014-I.

[65] U. Manthe, H.-D. Meyer, L. S. Cederbaum, Wave-packet dynamics within the multiconfiguration Hartree framework: General aspects and application to NOCl, J. Chem. Phys. 97 (1992) 3199. doi:http://dx.doi.org/10.1063/1.463007. 
[66] M. H. Beck, A. Jäckle, G. A. Worth, H.-D. Meyer, The multiconfiguration time-dependent Hartree (MCTDH) method: a highly efficient algorithm for propagating wavepackets, Phys. Rep. 324 (2000) 1. doi:http://dx.doi.org/10.1016/S0370-1573(99)00047-2.

[67] H.-D. Meyer, F. Gatti, G. A. Worth (Eds.), Multidimensional Quantum Dynamics, Wiley-VCH, 2009.

[68] A. Jäckle, H.-D. Meyer, Product representation of potential energy surfaces, J. Chem. Phys. 104 (1996) 7974. doi:http://dx.doi.org/10.1063/1.471513.

[69] A. Jäckle, H.-D. Meyer, Product representation of potential energy surfaces ii, J. Chem. Phys. 109 (1998) 3772. doi:http://dx.doi.org/10.1063/1.476977.

[70] Y.-C. Chiang, P. V. Demekhin, A. I. Kuleff, S. Scheit, L. S. Cederbaum, Linewidth and lifetime of atomic levels and the time evolution of spectra and coincidence spectra, Phys. Rev. A. 81 (2010) 032511. doi:http://dx.doi.org/10.1103/PhysRevA.81.032511.

[71] P. V. Demekhin, L. S. Cederbaum, Strong interference effects in the resonant Auger decay of atoms induced by intense x-ray fields, Phys. Rev. A. 83 (2011) 023422. doi:http://dx.doi.org/10.1103/PhysRevA.83.023422.

[72] E. Pahl, H.-D. Meyer, L. S. Cederbaum, Competition between excitation and electronic decay of short-lived molecular states, Z. Phys. D 38 (1996) 215. doi:http://dx.doi.org/10.1007/s004600050086. 
[73] Y. Berlatzky, S. Klaiman, Controlling Fano profiles via conical intersections, Phys. Rev. B 79 (2009) 085303. doi:10.1103/PhysRevB.79.085303.

[74] A. C. Johnson, C. M. Marcus, M. P. Hanson, A. C. Gossard, Coulombmodified Fano resonance in a one-lead quantum dot, Phys. Rev. Lett. 93 (2004) 106803. doi:10.1103/PhysRevLett.93.106803. 